\title{
CHANGEMENT PÉTROGRAPHIQUE MAJEUR DANS LES HAUTES TERRASSES DE LA MEUSE ENTRE NAMUR ET HUY (BELGIQUE) À LA SUITE DE LA CAPTURE DE LA SEMOIS
}

\author{
Étienne JUVIGNÉ, Geoffrey HOUBRECHTS, Jean VAN CAMPENHOUT
}

\begin{abstract}
Résumé
Une étude sédimentologique de lambeaux de très hautes terrasses mosanes entre Namur et Huy a permis d'améliorer les connaissances relatives à l'évolution de la Meuse de Dinant qui a façonné des terrasses sur $35 \mathrm{~m}$ de dénivelée sous le niveau de la transgression oligocène du plateau en y abandonnant systématiquement un cailloutis épais de quelques décimètres et caractérisé par une large majorité de galets de quartz ainsi qu'une granularité essentiellement inférieure à $32 \mathrm{~mm}$. À l'altitude actuelle de $180 \mathrm{~m}$ à Andenne/Huy, un cailloutis grossier et riche en cailloux roulés de quartzite a brutalement envahi le bassin ; il est attribué à la capture de la Semois survenue à Revin (France). Cette capture est placée dans le Pléistocène inférieur sur la base de sa position stratigraphique dans les modèles de terrasse existants, plutôt que dans le Mio-Pliocène comme évoqué dans la littérature.
\end{abstract}

Mots-clefs

Belgique, Namur, Huy, Meuse, Semois, capture, terrasse, sédimentologie

\begin{abstract}
This sedimentological study focusing on remnants of very high terraces of the Meuse River between Namur and Huy allowed improving the knowledge related to the evolution of the so-called "Meuse of Dinant". The fluvial terraces spanning a height difference of $35 \mathrm{~m}$ below the plateau level shaped by the Oligocene transgression are systematically characterized by a thin gravel layer mostly composed of quartz pebbles essentially lower than $32 \mathrm{~mm}$ in size. At the current altitude of $180 \mathrm{~m}$ at Andenne/Huy, a coarse, quartzite-rich gravel suddenly occurred in the basin; it is attributed to the capture of the Semois at Revin (France). This capture is assigned to the Lower Pleistocene based on its stratigraphic position in the existing terrace models instead of the Mio-Pliocene as previously thought.
\end{abstract}

Keywords

Belgium, Namur, Huy, Meuse, Semois, capture, terrace, sedimentology

\section{INTRODUCTION}

Des aspects de l'évolution du cours de la Meuse entre Namur et Huy ont été abordés par Stainier (1894, 1926), Fourmarier (1905, 1924), Mouchamps (1927, 1933), Clairbois (1957, 1959), Dewez (1998), Juvigné et al. (2013, 2015). Des modèles de terrasse de la Meuse depuis la France jusqu'au Limbourg ont aussi intégré des résultats acquis par des auteurs précités (Rigo, 1935 ; Bestels, 1949 ; Pissart, 1975 ; Pissart et al., 1997) ; toutefois, ils sont basés essentiellement sur des raccords géométriques de proche en proche de replats portant du gravier fluviatile, mais aussi d'autres moins significatifs, sans gravier comme des lambeaux d'aplanissement partiel ou des terrasses supposées dénudées.
Les travaux de synthèse les plus récents (Pissart, 1975 ; Pissart et al., 1997 ; Rixhon et Demoulin, 2018) traitant du cours de la Meuse depuis la Lorraine (France) jusqu'au Limbourg (Belgique et Pays-Bas) intègrent dans leurs modèles respectifs des résultats acquis par tronçon par des auteurs différents. Or sur la plus grande partie du cours depuis l'entrée en Belgique jusqu'à Liège, le seul modèle disponible relève d'un travail de fin d'études (Clairbois, 1957) dont aucune partie n'a jamais fait l'objet d'études complémentaires.

Un outil stratigraphique évoqué par Stainier (1894) a été négligé dans tous les travaux précités. Il s'agit d'une différence pétrographique majeure propre aux cailloutis que l'auteur a groupé en deux catégories, d'une part les « Amas de cailloux blancs des hauts 
plateaux (Tertiaire) » et d'autre part les « Amas de gravier des terrasses supérieures (Quaternaire $q 2 m)$ ». Il situe la transition de l'un à l'autre aux environs de $175 \mathrm{~m}$ d'altitude sur substratum siliceux ou schisteux dans la région de Huy, et il fait remarquer que sur des roches calcaires et/ou dolomitiques, il faut s'attendre à ce que les dépôts fluviatiles se trouvent en position effondrée.

La distinction pétrographique précitée apparaît également dans la légende de la carte géologique ancienne (fin du XIX et début du XX $\mathrm{XX}^{\mathrm{e}}$ siècles) : (1) le label Onx a été attribué aux dépôts de cailloutis qui tapissent, entre autres, le plateau de Hesbaye et sa retombée méridionale ; il répond à la définition "Amas et traînées de cailloux blancs à allures ravinantes et fluviales », et il est placé dans l'Oligocène supérieur, dépôts continentaux ; (2) le label $q 2 m$ est défini comme «Cailloux des terrasses moyennes des grandes vallées »; il désigne les cailloutis mosans nettement plus grossiers et plus riches en quartzite et grès divers qui sont postérieurs aux Onx. En 1933, au terme d'une polémique longue de trois décennies sur l'âge de ces cailloutis (voir par exemple : Van den Broeck et Rutot, 1888a ; Van den Broeck, 1889 ; Stainier, 1894 ; Lohest, 1896 ; Forir, 1897-1898 ; Briquet, 1907, 1922 ; Rutot, 1908 ; Bellière, 1924), le Conseil géologique de Belgique en a décidé le transfert dans le Pliocène final. Dès lors, le label Onx impliquant un âge oligocène devenait de fait obsolète; néanmoins nous en faisons usage non seulement dans les évocations d'auteurs, mais aussi parce que la légende de la nouvelle carte géologique sous le label Ala (Alluvions anciennes), ne fait malheureusement plus la distinction entre ces deux types de cailloutis pourtant si différents.
Le but du présent travail vise à apporter des résultats d'analyses sédimentologiques de lambeaux de terrasses fluviales (lbxt; au singulier lbt) revisités entre Namur et Huy (Figure 1) et de vérifier la compatibilité des résultats avec le modèle original et les synthèses, établis par la seule méthode évoquée plus haut.

Organisation du travail. On trouve dans l'ordre: (a) les méthodes appliquées ; (b) la description de sites de référence; (c) un renvoi au rapport de recherche (Juvigné et al., 2020a) pour la description aussi détaillée des autres sites revisités ; (d) un tableau reprenant les caractéristiques utiles de tous les sites revisités ; (e) des données sédimentologiques nouvelles présentées et discutées globalement; (f) une discussion confrontant les résultats nouveaux avec ceux de la littérature.

\section{MÉTHODES}

Prélèvements d'échantillons. Pour chaque site, au moins deux échantillons d'environ $10 \mathrm{dm}^{3}$ (un seau) ont été prélevés : (1) autant que possible en affleurement avec entre eux une distance faible de l'ordre du mètre, voire du décamètre (carrière, talus); (2) aux deux extrémités d'une tranchée artisanale jusqu'à $60 \mathrm{~cm}$ de profondeur (sub-surface) ; (3) dans les mêmes conditions à des distances hectométriques sur un replat d'altitude constante ; (4) au moyen d'une pelle mécanique à une profondeur maximale de $2 \mathrm{~m}$ dans des sites d'intérêt particulier.

La distribution granulométrique. Mode opératoire : (1) dispersion de l'échantillon dans l'eau et tamisage à $2 \mathrm{~mm}$ sous courant d'eau jusqu'à obtenir

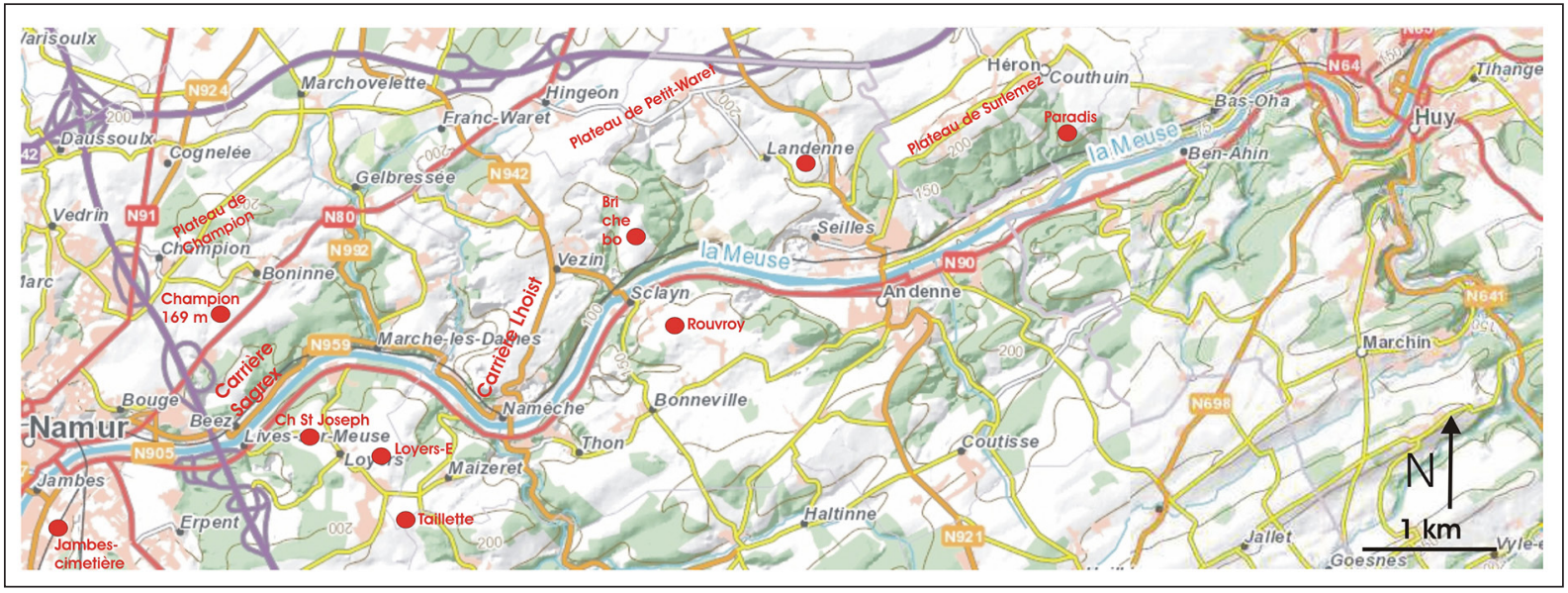

Figure 1. Localisation des sites revisités (Fond de carte extrait du site WalOnMap) 

entre Namur et Huy (Belgique) à la suite de la capture de la Semois

une récolte de cailloux propres ; (2) séchage ; (3) tamisage à sec sur une colonne de tamis avec pas de phi/2 ; (4) représentation de la granularité des échantillons en histogrammes dans lesquels, pour un lbt déterminé, la courbe granulométrique moyenne pondérée est ajoutée.

Nature, forme et faciès des cailloux. Dans l'ordre de la taille à partir de la classe la plus grossière, celle de 8 à $16 \mathrm{~mm}$ est la première qui, dans les conditions de prélèvement précitées, contient suffisamment d'éléments pour soutenir les valeurs calculées. Lors de la répartition des éléments en classes, il est apparu que pour distinguer les graviers $O n x$ et $q 2 m$, on pouvait se limiter à des critères qui ne laissent que peu de place aux erreurs de détermination. En matière de nature et de faciès des cailloux, les indices sont appliqués à la seule fraction de 8 à $16 \mathrm{~mm}$, et ils ont été conçus en prenant pour modèle celui qui a été introduit par Van Straaten $(1946,1947)$ pour les graviers mosans du Limbourg néerlandais, à savoir sous une forme générique : $K^{\prime}=100^{*} A /(A+B)$; il varie de 0 à 100 , et A est le facteur que l'on souhaite mettre en évidence. Dans l'indice de fréquence des quartz, A est le nombre de quartz purs et B l'ensemble des cailloux émoussés entiers ou cassés ; la formule ignore donc les cailloux qui n'ont pas subi d'usure perceptible, c'est-à-dire en principe les éléments locaux, comme par exemple des cailloux à arêtes uniquement vives issus directement du socle local, des débris de croûte de fer-manganèse, des silex bruts.... Dans l'indice de polissage des quartz, A est le nombre de quartz entiers bien polis, ou cassés dont une partie atteste qu'ils étaient bien polis. En ce qui concerne l'indice d'arrondi, seuls les quartz ont été soumis au traitement informatique d'image suivant la méthode de Roussillon et al. (2009), et de tous les indices proposés par ces auteurs, l'indice d'arrondi de Wadell (Iw) est apparu le plus discriminant pour la distinction entre $O n x$ et $q 2 m$. Bien que le thème de ce travail soit limité aux plus hautes terrasses, des déterminations ont été effectuées pour des terrasses moyennes et basses de façon à mettre en évidence la tendance générale dans toute la série de terrasses mosanes.

Minéraux denses transparents (Mdt). Les recherches ont été concentrées sur la fraction comprise entre 75 et $425 \mu \mathrm{m}$ et les déterminations effectuées en trois classes granulométriques ; 75-150 $\mu \mathrm{m} ; 150$ $300 \mu \mathrm{m} ;>300 \mu \mathrm{m}$. L'élimination de la fraction inférieure à $75 \mu \mathrm{m}$ a été voulue notamment pour éviter les minéraux des lœss nécessairement présents dans la matrice des cailloutis. Toutefois, par le fait même, la plupart des minéraux des altérites du socle paléozoïque (en général des ubiquistes provenant de roche à texture limono-argileuse) ont évidemment été éliminés. Par contre, les sables marins tertiaires sont bien représentés dans les associations des sables fluviatiles.

Le travail de référence en matière de terrasses mosanes sur le tronçon étudié est inédit (Clairbois, 1957); il a conduit à un résumé publié (Clairbois, 1959). C'est évidemment dans le premier travail que les renseignements détaillés peuvent être trouvés. Toutefois, en matière de labels de terrasses, il faut noter que dans le travail original, les trois plus hautes terrasses, sont appelées T8, T9' et T9 de la plus récente à la plus ancienne, tandis que dans la publication, les labels des mêmes terrasses sont devenus respectivement T8, T9 et T10. Par souci de cohérence, les labels du travail original sont utilisés plus bas.

Documents cartographiques. La carte topographique de Belgique (IGM, 1903) a été le plus souvent préférée à la carte la plus récente parce qu'elle présente les avantages d'une faible urbanisation et d'une équidistance qui descend jusqu'à 1 m. Néanmoins, le réseau PICC ou le GPS ont été utilisés lorsqu'une précision inférieure au mètre était requise.

Archives. Les archives du Service géologique de Belgique ont permis de récolter le plus grand nombre d'informations possibles sur les lbxt.

\section{LOCALISATION, DESCRIPTION ET ALTITUDE DE QUELQUES DÉPÔTS DE CAILLOUTIS ONX ET DES PREMIERS CAILLOUTIS Q2M}

Pour chacun des deux types de cailloutis distingués par Stainier (op. cit.), nous avons choisi deux sites de référence proches l'un de l'autre sur le territoire de la commune de Couthuin : (1) la butte de Surlemez (219 m); (2) un vaste replat au lieu-dit Paradis $(180 \mathrm{~m})$ (Figure 2). Dans les deux cas, le socle paléozoïque consiste essentiellement en schistes, grès et psammites du Houiller. Il n'y a donc pas de possibilité d'effondrement des cailloutis par karstification. 


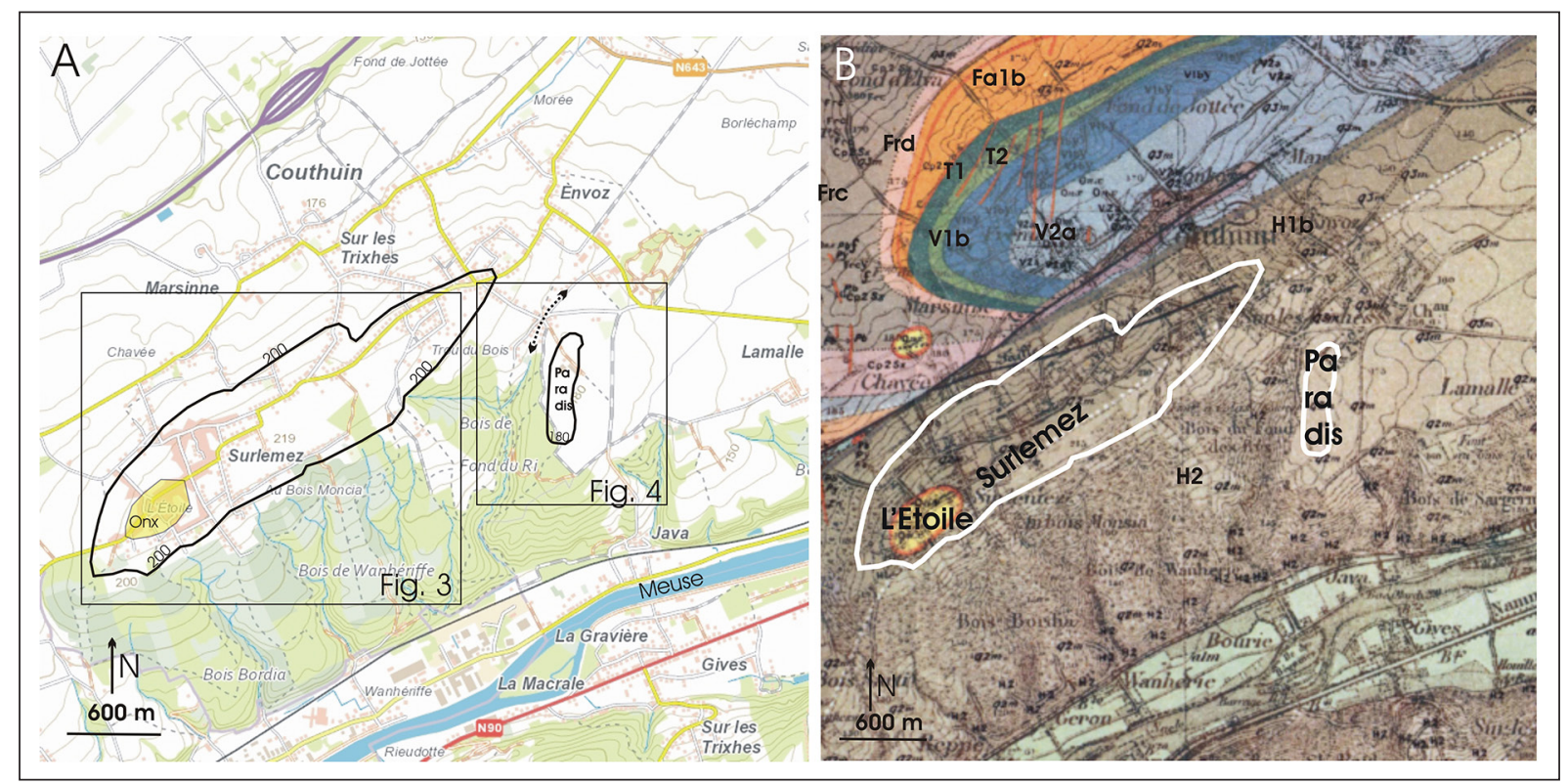

Figure 2. La butte de Surlemez et le replat de Paradis (A) Localisation sur la carte topographique. Explications : trait interrompu bi-fléché= ensellement qui met le replat de Paradis en inversion de relief ; (B) Extrait de la carte géologique ancienne (Stainier, 1901a); lithologie : H2= grès psammites, schistes, houilles variées ; H1b=grès, psammites, schistes, houilles maigres ; V2a=calcaires ; V1b= grandes dolomies ; $\mathrm{T} 2=$ dolomies ; $\mathrm{T} 1=$ dolomies ; $\mathrm{Fa} 1 \mathrm{~b}=$ schistes avec psammites $; \mathrm{Frc}=$ calcaires noduleux $; \mathrm{Frd}=$ dolomies .

\section{A. Le plateau de Surlemez}

Le plateau de Surlemez s'étire suivant la direction SO-NE. Si l'on s'en tient à la zone où l'altitude est supérieure à $200 \mathrm{~m}$, la longueur est de $5 \mathrm{~km}$ et la largeur de $1 \mathrm{~km}$. Son point culminant est à $219 \mathrm{~m}$ (Figure 2).

\section{1. Évocation dans la littérature}

Stainier (1894) y voit des «Amas de cailloux blancs des hauts plateaux (Tertiaire) », d'une part au lieu-dit l'Étoile à $~ 215 \mathrm{~m}$ et d'autre part à Envoz à $\sim 210 \mathrm{~m}$. Sur la carte géologique (Stainier, 1901a), seul le cailloutis de L'Étoile est représenté (Figure 2B); l'extension du lbt est limitée à une dizaine d'hectares dans une zone où l'altitude du sol est un peu supérieure à $210 \mathrm{~m}$. La présence de cailloux Onx dans les sols autour de la butte est mentionnée. Lorié (1919) écrit que dans le village de Surlemez, le « Gravier blanc » affleure partout, et qu'entre Surlemez et Couthuin, il est « entremêlé, peu typique » avec des silex, des lydites et des grès. Meunier (1953) écrit sous l'intitulé « Surlemez», et sans autre précision : « Le cailloutis apparait dans les fossés qui bordent les chemins et à la surface des terres cultivées. \ Clairbois (1957) a vu une excavation à L'Étoile/Surlemez : « argile noirâtre, avec silex et cailloux roulés en petit nombre (ép. 1 m); dragées de quartz blanc...mélangée de cailloux de plus gros calibre (ép. $1 \mathrm{~m}$ ); éléments nucléaires (NDR : comprendre nuculaire $=$ taille de la noix) indéterminés ; base du dépôt non visible ». Elle rapporte également qu'à $10 \mathrm{~m}$ au nord, dans un puits, le schiste a été atteint. Sur le replat, elle a vu dans le sol labouré, « un maximum de dragées entre 210 et $215 \mathrm{~m} »$. En conséquence, l'auteur considère l'ensemble de la butte de Surlemez comme un seul lambeau dont la base du cailloutis est placée à $210 \mathrm{~m}$, et la terrasse mosane correspondante porte le label T9, défini comme «...tous les dépôts qui possèdent les caractères propres à la Traînée mosane : dragées de quartz blanc, dragées roses, kieseloolithes. »

\section{Nouvelles données de terrain}

Deux documents d'archives du Service géologique très anciens n'ont jamais été cités dans la littérature relative au cailloutis qui nous occupe (Figure 3 ) : (1) SvG332 est un forage carotté réalisé en 1938 pour la commune de Couthuin à $218 \mathrm{~m}$ d'altitude (déterminée par nous a posteriori); il a été décrit par un auteur anonyme qui au terme de son rapport détaillé conclut à la présence de « limons $1 \mathrm{~m} 75$; graviers (Onx) $2 \mathrm{~m} 75$; dépôts continentaux (Om) 6m50». Ces derniers sont en général argileux, et leur base est plus basse que $207 \mathrm{~m}$; (2) SvG270 est à l'altitude 


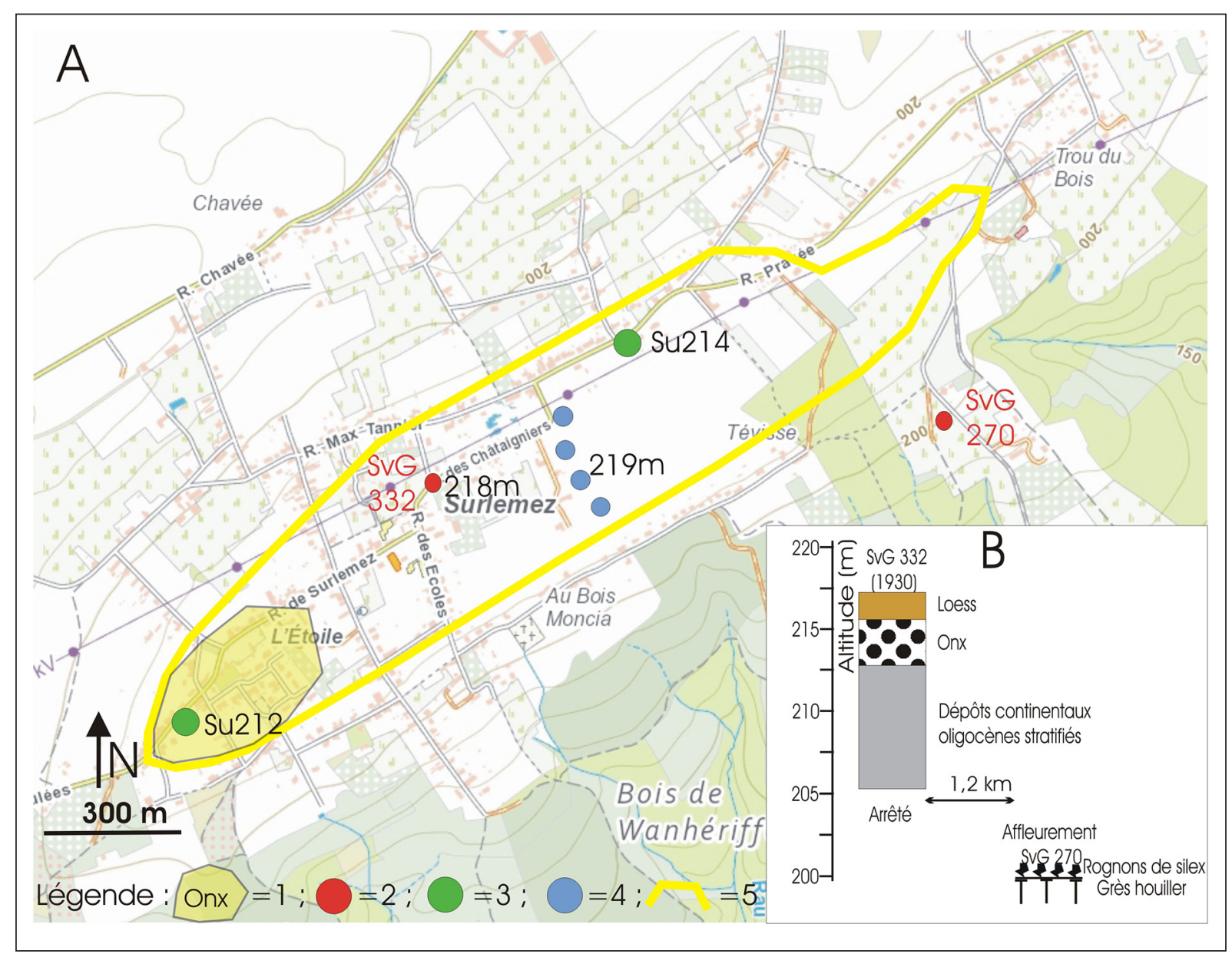

Figure 3. La butte de Surlemez. (A) Extension et sites d'intérêt particulier. Légende : 1= le lbt d'Onx d'après la carte géologique (Stainier, 1901) ; 2= forage carotté et affleurement (archives du Service géologique de Belgique) ; $3=$ localisation des échantillons de cailloutis ; $4=$ forages personnels à la tarière jusqu'au toit du cailloutis ; 5= limite d'extension proposée pour le cailloutis en position primaire correspondant à l'altitude probable de sa base (courbe de niveau de $213 \mathrm{~m}$ ). (B) Position altimétrique de la colonne lithostratigraphique du forage SvG332 et de l'affleurement SvG270.

de $200 \mathrm{~m}$ « affleurement direct de cailloux blancs, avec beaucoup de gros rognons et de fragments de silex. Ces silex sont évidemment sous l'Oligocène, et ils reposent sur le grès houiller. ॥ (Rutot, 1900). Le contact des dépôts continentaux oligocènes avec le socle houiller au point SvG332 devrait donc se trouver entre 200 et $207 \mathrm{~m}$. Nous avons exécuté des forages à la tarière sur la crête de la butte, et le toit du cailloutis y a été atteint dans tous les cas entre 215,5 et $216,5 \mathrm{~m}$. Le cailloutis Onx est donc compris entre $213 \mathrm{~m}$ (base) et $216,5 \mathrm{~m}$ (sommet) et son épaisseur maximale connue $(2,75 \mathrm{~m})$ a été mesurée dans le forage SvG232.

Deux échantillons ont été prélevés : (1) Su214 provient d'un cailloutis rubéfié qui a été vu sous $2 \mathrm{~m}$ de loess dans le fond d'une excavation qui avait déjà reçu les caves d'une maison en construction (rue Max Tannier $n^{\circ} 13 B$ ) ; il a été prélevé sur une ving- taine de centimètres d'épaisseur à $214 \mathrm{~m}$ d'altitude ; (2) $\mathrm{Su} 212$ provient d'un affleurement sur un talus routier au lieu-dit L'Étoile à 212 m d'altitude dans une position marginale du lambeau, et probablement $\sim 1 \mathrm{~m}$ sous le niveau de la base déterminée plus haut.

\section{Synthèse}

Dans la butte de Surlemez, la nappe de cailloutis doit se trouver en position primaire entre $213 \mathrm{~m}$ (base) et $216,5 \mathrm{~m}$ (sommet) et son épaisseur maximale mesurée en un même point est de $2,75 \mathrm{~m}$. Le cailloutis est classé dans 1'Onx sur la carte géologique. Il repose sur des dépôts continentaux oligocènes, en général argileux. Le socle est constitué de roches siliceuses et argileuses, ce qui exclut les déformations karstiques. Les précisions apportées sur l'altitude du gravier nous conduisent de proposer au lbt un label spécifique : Cou-Sur[b213; s216 ; d138]. 


\section{B. Le replat de Paradis à Couthuin}

Il s'agit du lbt occupé par la seule ferme Wéra au lieu-dit Paradis. Le replat en inversion de relief culmine entre $\sim 180$ et $181 \mathrm{~m}$ (Figure 4) au pied méridional de la partie nord-est du plateau de Surlemez (Figure 2).

\section{1. Évocation dans la littérature}

Stainier (1894) cartographie le lambeau et l'attribue aux « Amas de graviers des terrasses supérieures (Quaternaire $q 2 m$ )». Clairbois (1957) cartographie ce replat sous le $n^{\circ} 81$ et écrit : "Son immense surface absolument plane, est couverte de cailloux roulés en assez grand nombre, dont la base se situe vers 180 mètres. »

\section{Nouvelles données de terrain}

Si l'on considère la partie plus élevée que 179 m, le replat de Paradis est long d'environ $500 \mathrm{~m}$ et

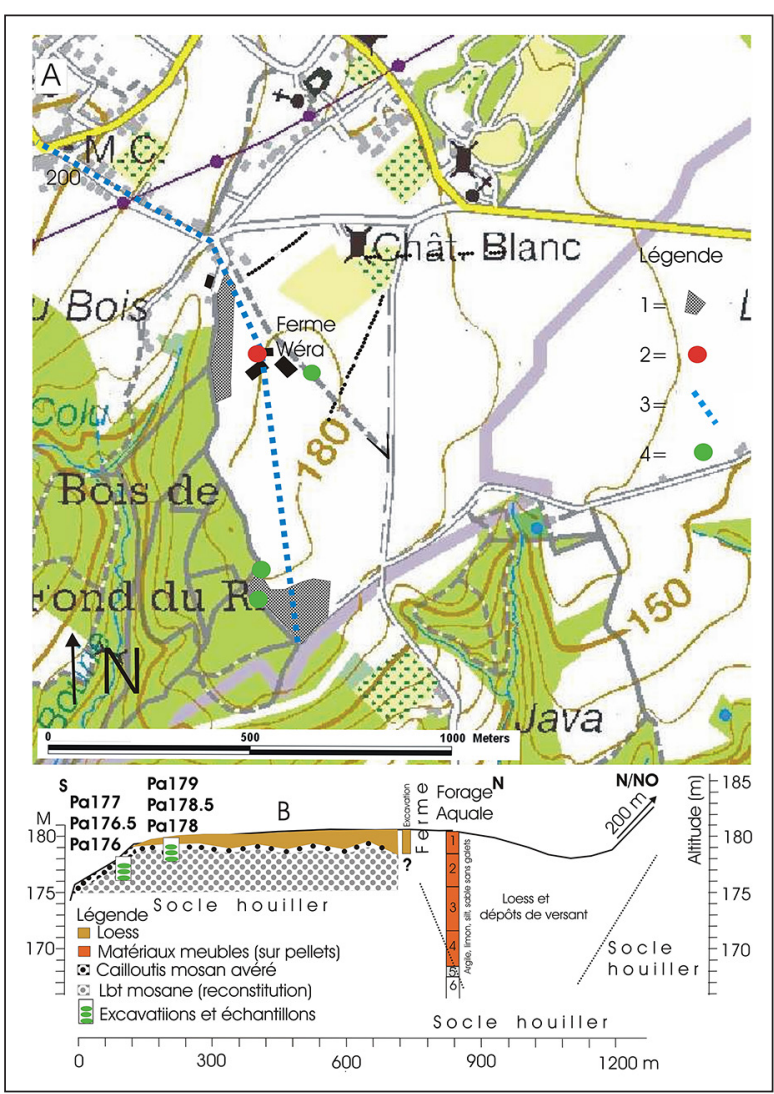

Figure 4. Le lbt de Paradis/Couthuin (voir la localisation sur la figure 2). (A) Relief détaillé et sites particuliers. Explications : $1=$ cailloux roulés abondant dans le sol labouré ; $2=$ forage industriel profond réalisé par la firme Aquale ; $3=$ position de la coupe de la figure B ; $4=$ position des excavations pour le prélèvement des échantillons (B) Coupe longitudinale dans le lbt. large de $300 \mathrm{~m}$; il n'atteint pas $181 \mathrm{~m}$ d'altitude (Figure 4A). Un ensellement l'isole de la butte de Surlemez, en conséquence de l'érosion régressive de deux ruisseaux divergents dont les têtes de vallée sont dos à dos et coalescentes (Figure 2).

L'essentiel du matériau en affleurement dans les terres labourées consiste en limon d'origine éolienne, mais dans deux zones des parties méridionale et occidentale du replat, la fréquence des cailloux dans les terres labourées est élevée. Le toit du schiste houiller est visible dans un talus à $175 \mathrm{~m}$ dans la partie méridionale.

Sur le replat, l'altitude du toit du cailloutis a été atteinte entre 178 et $179 \mathrm{~m}$ par une série de forages exécutés à la tarière à travers la couverture lœssique (Figure 4B), mais en bordure de la ferme Wéra, une excavation creusée à la mini-pelle jusqu'à $2 \mathrm{~m}$ de profondeur n'a mis au jour que du lœss, et un forage industriel (marteau fond de trou) a été réalisé par la Firme Aquale pour l'approvisionnement en eau, il a atteint le socle houiller à l'altitude de $166 \mathrm{~m}$ après avoir traversé des matériaux meubles identifiés sur pellets. Ce forage montre que l'ensellement fossile est beaucoup plus profond et abrupt au toit du socle houiller qu'en surface. Il a dû être creusé par l'érosion régressive des ruisseaux précités dans des conditions environnementales différentes des conditions actuelles. Des fouilles en tranchées ont été réalisées au moyen d'une pelle mécanique jusqu'à $2 \mathrm{~m}$ de profondeur dans le cailloutis de façon à disposer d'échantillons non affectés par des remaniements naturels (bio-, cryoturbations) et/ou anthropiques; ils portent les labels : Pa176; Pa176,5 ; Pa177 ; Pa178 ; Pa178,5 ; Pa179. Sous le sol labouré, le cailloutis est cryoturbé et intensément rubéfié.

\section{Synthèse}

Le cailloutis du lbt de Paradis est qualifié de $q 2 m$ sur l'ancienne carte géologique de Belgique (Stainier, 1901). Il est largement fossilisé sous une couverture lœssique. Sa partie supérieure est cryoturbée et rubéfiée. Il repose directement sur le socle siliceux du Houiller. Les précisions acquises sur la base et le sommet du cailloutis font de lui un lbt de référence dans l'évolution du cours de la Meuse ; un label spécifique lui est attribué : Cou-Par[b175; s179; d100] (Cou-Par= Couthuin-Paradis). 

entre Namur et Huy (Belgique) à la suite de la capture de la Semois

\section{La carrière Lhoist à Namèche}

La partie supérieure du front de taille de la carrière Lhoist à Namèche constitue un site de référence en matière de déformation de terrains de couverture qui reposent sur des roches solubles, ici des « Grandes dolomies » (V1b) et des calcaires (V2a). D'un point de vue morphologique, il s'agissait d'un plateau allongé SO/NE entre le ruisseau de Vezin et la Meuse entre Namèche et Sclaigneau. Avec pour limite la courbe de niveau de $170 \mathrm{~m}$, sa longueur est de $2 \mathrm{~km}$ et sa largeur $\sim 600 \mathrm{~m}$. Il porte deux points culminants respectivement à 182 et $183 \mathrm{~m}$ (Figure 5).

\section{1. Évocation dans la littérature}

À la fin du XIX ${ }^{\mathrm{e}}$ siècle, la carrière était limitée au versant abrupt de la vallée, et le plateau n'était pas excavé, si bien que le seul terrain de couverture représenté sur la carte géologique (Stainier, 1901b) au toit des « Grandes dolomies » du Viséen (V1by), est le « limon brunâtre et grisâtre stratifié' $(q 3 m)$ ». Clairbois (1957) écrit sans autre commentaire : «Le long replat (551) situé... entre 182 et 183 mètres représente un aplanissement partiel...».

\section{Nouvelles données de terrain}

La partie supérieure du front de taille est représentée à la Figure 6. Il s'y trouve une couverture de lœss qui atteint la dizaine de mètres d'épaisseur dans les parties les plus élevées du replat. Sous cette couverture se trouvent des sables limoneux rubéfiés qui colmatent un réseau de poches de dolomie karstifiée dont la taille est décamétrique. Les colonnes et le fond des poches sont tapissés par une couche d'épaisseur pluri-décimétrique de cailloutis de type Onx. Cet ensemble a une épaisseur de 6 à $8 \mathrm{~m}$. Les colonnes de dolomie sont tronquées à $171 \mathrm{~m}$, et l'altitude du plancher des poches varie entre 160 et $164 \mathrm{~m}$. La cote de $171 \mathrm{~m}$ est donc une altitude minimale pour la position originelle du cailloutis. Le replat n'est donc pas un lambeau d'aplanissement partiel à $180 \mathrm{~m}$ ( $c f$. supra), mais plutôt la surface d'une accumulation lœssique.

Les têtes de colonnes de dolomie tronquées à $171 \mathrm{~m}$ représentent les restes d'une surface d'érosion façonnée par un ruissellement en nappe qui remaniait des galets Onx provenant du plateau hesbignon (Petit-Waret et/ou Wartet). Compte tenu de la mise en

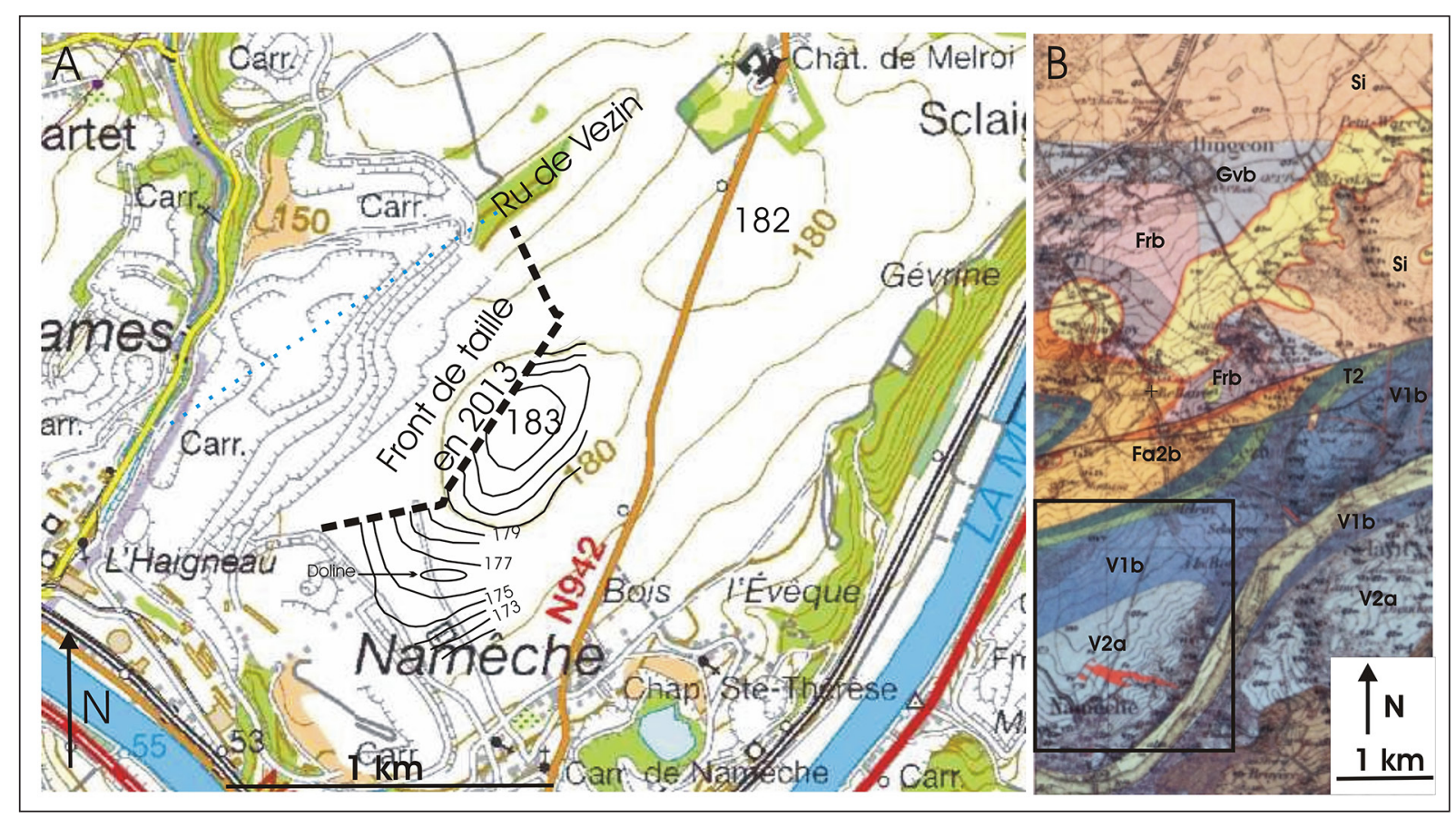

Figure 5. La carrière des 'Dolomies de Marches-les-Dames' à Namèche. (A) Localisation du front de taille décrit dans ce travail (trait gras interrompu) et ajout de courbes de niveau (trait noirs fins) pour préciser l'altitude du cimetière où le cailloutis Onx est régulièrement excavé. (B) Extrait de la carte géologique (Stainier, 1901b); lithologie : $\mathrm{V} 1 \mathrm{~b}=$ grandes dolomies ; $\mathrm{V} 2 \mathrm{a}=$ calcaires $; \mathrm{T} 2=$ dolomie ; Fa2b, roches siliceuses ; Frasnien $(\mathrm{Fr}$, diverses roches carbonatées) ; Givetien (les couches givetiennes dans cette partie du bassin sont essentiellement gréso-schisteuses et ne renferment plus que des intercalations calcaires de faible puissance, souvent salies de matériaux détritiques, peu propices à la karstification (Delcambre, en charge de la révision de la carte géologique ; communication écrite); Silurien (Si2b, schistes). 
place de la nappe Onx à $171 \mathrm{~m}$, il ne peut s'agir d'un lbt en position primaire puisque le cailloutis $q 2 \mathrm{~m}$ était déjà présent à Paradis entre 175 et $179 \mathrm{~m}$ (voir plus haut). La surface d'érosion de Namèche devait donc se raccorder à une terrasse mosane inférieure à celle de Paradis et située à l'aplomb de la vallée actuelle ou de son flanc droit. Au fur et à mesure de la karstification, le cailloutis s'est effondré et du sable limoneux probablement éolien a colmaté les poches, le tout dans un environnement chaud et humide provoquant une intense rubéfaction des matériaux de remplissage. Deux échantillons de cailloutis ont été prélevés sur des têtes de colonnes de dolomie de façon à éviter autant que possible les cailloux de dolomie qui ont été entraînés avec le cailloutis dans le fond des poches karstiques ; ils portent les labels Na171a et Na171b.

\section{Synthèse}

Le replat de Namèche à $180 \mathrm{~m}$ est une surface d'accumulation lœssique. Sous le lœss se trouve un cailloutis $O n x$ perturbé par la karstification qui constitue un site de référence permettant d'imaginer la façon dont se présentent les dépôts de couverture se trouvant sur d'autres replats dont le substratum est constitué de roches paléozoïques solubles (calcaires et dolomies).

Rappel : tous les autres sites revisités (revoir la Figure 1) sont décrits de façon aussi détaillée que les sites de référence dans Juvigné et al. (2020a).

\section{SÉDIMENTOLOGIE : ONX VS Q2M, IN- DICES DISCRIMINATOIRES}

Les résultats essentiels propres aux différents lbxt revisités sont rassemblés dans le Tableau 1. Les échantillons y sont classés dans l'ordre d'altitude du point de prélèvement, mais cette relation n'est qu'indicative pour deux raisons majeures : (1) des échantillons sont à l'aplomb de roches calcaires karstifiées ; (2) les mêmes et/ou d'autres sont en position remaniée sur des versants dont le socle
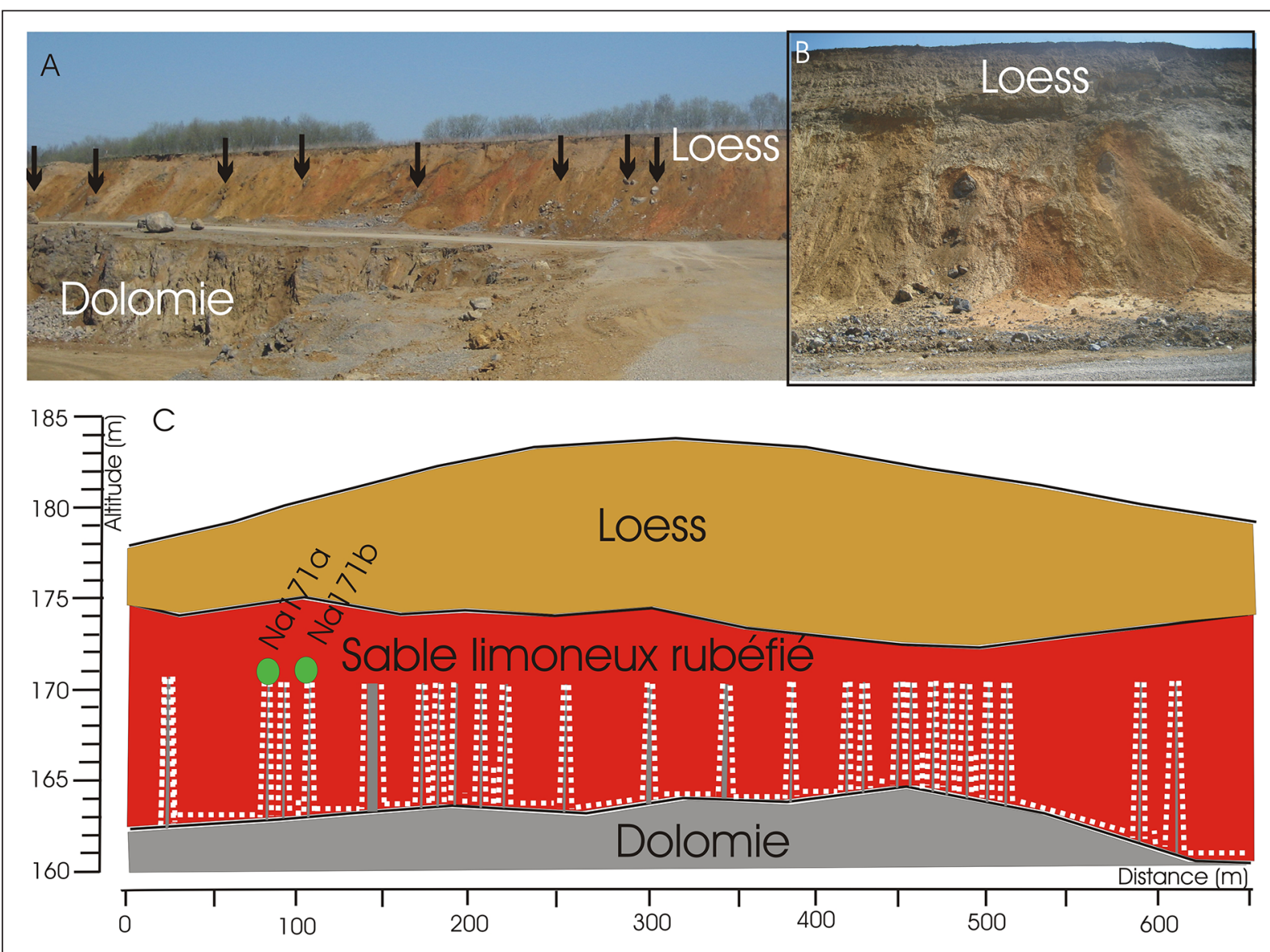

Figure 6. Les terrains de couverture de la carrière Lhoist à Namèche. (A) Le front de taille supérieur en 2019. Entre le lœss et la dolomie se trouve le niveau de dolomie karstifiée avec colonnes résiduelles et dépôts de remplissage ; les flèches noires montrent des colonnes de dolomie. (B) Gros plan d'une colonne de dolomie tapissée de cailloutis. (C) Coupe des terrains de couverture. Explications : colonnes grises= colonnes de dolomie ; les points blancs représentent le cailloutis qui tapisse la dolomie karstifiée ; pastilles vertes= position des échantillons de cailloutis Na171a et Na171b. 
Changement pétrographique majeur dans les hautes terrasses de la Meuse entre Namur et Huy (Belgique) à la suite de la capture de la Semois

\begin{tabular}{|c|c|c|c|c|c|c|c|c|c|c|c|c|c|c|c|c|c|c|c|}
\hline \multirow{2}{*}{$n^{\circ}$} & \multirow{2}{*}{ Echantillons } & \multicolumn{6}{|c|}{ Données de terrain } & \multirow[b]{2}{*}{$\mathrm{N}^{\circ} \mathrm{ds}$} & \multicolumn{6}{|c|}{ Indices de nature et de forme } & \multicolumn{5}{|c|}{ Indices granulométriques } \\
\hline & & $\operatorname{Lb} X$ & Lb Y & Base & Toit & Epais & Socle & & $\mathrm{n}=$ & Iw & $\mathrm{n}=$ & $Q^{\prime}$ & $\mathrm{G}^{\prime}$ & $\mathrm{Ca} \%$ & Mo1 & Mo2 & Mé & Q3 & + gros \\
\hline \multicolumn{3}{|c|}{ Onx de plateau (213-217 m) } & & & & & & Clair. & & & & & & & & & & & \\
\hline 1 & Su214 & $50^{\circ} 31^{\prime} 19.95^{\prime \prime} \mathrm{N}$ & $5^{\circ} 07^{\prime} 25^{\prime} .00^{\prime \prime} \mathrm{E}$ & 213 & 216,5 & $3,5 \mathrm{~m}$ & $S \& A$ & 1229 & 112 & 0,749 & 476 & 85,3 & 82 & 0 & 9,5 & & 6,7 & 9,5 & 32 \\
\hline 2 & Su212 & $50^{\circ} 30^{\prime} 51.20^{\prime \prime} \mathrm{N}$ & $5^{\circ} 06^{\prime} 26.58^{\prime \prime E}$ & 213 & 216,5 & $3,5 \mathrm{~m}$ & $S \& A$ & 1229 & 109 & 0,774 & 240 & 88,8 & 91,5 & 0 & & & & & \\
\hline 3 & PW210 & $50^{\circ} 31^{\prime} 23.74^{\prime \prime} \mathrm{N}$ & $5^{\circ} 01^{\prime} 24.42^{\prime \prime} \mathrm{E}$ & 213 & 216 & $3 m$ & $S \& A$ & 105 & 91 & 0,761 & 326 & 78,8 & 93,7 & 1 & 13,5 & & 13,5 & 19 & 45,3 \\
\hline 4 & PW206 & $50^{\circ} 31^{\prime} 31.00^{\prime \prime} \mathrm{N}$ & $5^{\circ} 02^{\prime} 13.36^{\prime \prime E}$ & 213 & 216 & $3 m$ & $S \& A$ & 105 & 83 & 0,747 & 463 & 78,7 & 88,5 & 0,2 & & & & & \\
\hline 5 & Ch208a & $50^{\circ} 30^{\prime} 07.03^{\prime \prime} \mathrm{N}$ & $4^{\circ} 55^{\prime} 29.52^{\prime \prime} \mathrm{E}$ & 213 & 216 & & Ca-Do & 524 & 131 & 0,741 & 283 & 78,6 & 95,5 & 2 & 13,5 & & 9,5 & 13,5 & 45,3 \\
\hline \multirow[t]{3}{*}{6} & Ch208b & $50^{\circ} 30^{\prime} 07.03^{\prime \prime} \mathrm{N}$ & $4^{\circ} 55^{\prime} 29.52^{\prime \prime E}$ & 213 & 216 & & Ca-Do & 524 & 137 & 0,757 & 253 & 81,4 & 96 & 1 & & & & & \\
\hline & Moyenne & & & & & & & & 111 & 0,755 & 340 & 81,9 & 91,2 & 0,7 & 12,2 & & 9,9 & 14 & 40,87 \\
\hline & Onx de replats & & & & & & & & & & & & & & & & & & \\
\hline 7 & Lo198a & $50^{\circ} 27^{\prime} 05.43^{\prime \prime} \mathrm{N}$ & $4^{\circ} 57^{\prime} 52.06^{\prime \prime} \mathrm{E}$ & 197 & 198 & $1 \mathrm{~m}$ & $S \& A$ & Illis. & 113 & 0,763 & 293 & 73,1 & 91,9 & 3,8 & 13,5 & 26,9 & 13,5 & 26,9 & 45,3 \\
\hline 8 & Lo198b & $50^{\circ} 27^{\prime} 05.43^{\prime \prime} \mathrm{N}$ & $4^{\circ} 57^{\prime} 52.06^{\prime \prime} \mathrm{E}$ & 197 & 198 & $1 \mathrm{~m}$ & $S \& A$ & Illis. & 105 & 0,754 & 176 & 70,9 & 89,3 & 1,7 & & & & & \\
\hline 9 & Br186a & $50^{\circ} 30^{\prime} 09.98^{\prime \prime} \mathrm{N}$ & $5^{\circ} 01^{\prime} 45.24^{\prime \prime E}$ & 184,5 & 186 & $1,5 \mathrm{~m}$ & Ca-Do & Illis. & 98 & 0,752 & 180 & 74,6 & 84,8 & 0 & 13,5 & & 13,5 & 19 & 45 \\
\hline 10 & Br186b & $50^{\circ} 30^{\prime} 09.98^{\prime \prime} \mathrm{N}$ & $5^{\circ} 01^{\prime} 45.24^{\prime \prime} \mathrm{E}$ & 184,5 & 186 & $1,5 \mathrm{~m}$ & Ca-Do & Illis. & 83 & 0,733 & 224 & 83,4 & 93,5 & 0 & & & & & \\
\hline 11 & Lo184a & $50^{\circ} 27^{\prime} 37.43^{\prime \prime} \mathrm{N}$ & $4^{\circ} 57^{\prime} 37.43^{\prime \prime} \mathrm{E}$ & \multicolumn{2}{|c|}{ s.o. } & $X \mathrm{dm}$ & Ca-Do & Illis. & 112 & 0,741 & 167 & 76,8 & 74,6 & 0,6 & 13,5 & 26,9 & 13,5 & 19 & 45,3 \\
\hline 12 & Lo184b & $50^{\circ} 27^{\prime} 37.43^{\prime \prime} \mathrm{N}$ & $4^{\circ} 57^{\prime} 37.43^{\prime \prime} \mathrm{E}$ & \multicolumn{2}{|c|}{ s.o. } & $x d m$ & Ca-Do & Illis. & 123 & 0,74 & 245 & 68,8 & 89,4 & 0,8 & & & & & \\
\hline 13 & La183a & $50^{\circ} 31^{\prime} 06.17^{\prime \prime} \mathrm{N}$ & 503'57.83"E & \multicolumn{2}{|c|}{ s.o. } & $x \mathrm{dm}$ & $S \& A$ & 325 & 91 & 0,762 & 283 & 87,1 & 90,6 & 0 & 19 & & 19 & 19 & 45,3 \\
\hline 14 & La183b & $50^{\circ} 31^{\prime} 06.17^{\prime \prime} \mathrm{N}$ & $5^{\circ} 03^{\prime} 57.83^{\prime \prime E}$ & \multicolumn{2}{|c|}{ s.o. } & $x \mathrm{dm}$ & $S \& A$ & 325 & 92 & 0,764 & 220 & 81,6 & 90,7 & 0 & & & & & \\
\hline 15 & La177 & $50^{\circ} 30^{\prime} 57.65^{\prime \prime} \mathrm{N}$ & $5^{\circ} 03^{\prime} 58.23^{\prime \prime} \mathrm{E}$ & \multicolumn{2}{|c|}{ s.o. } & $x d m$ & $S \& A$ & Néant & 45 & 0,738 & 111 & 50,5 & 91,3 & 0 & 26,9 & & 19 & 26,9 & 64 \\
\hline 16 & La175 & $50^{\circ} 30^{\prime} 57.65^{\prime \prime} \mathrm{N}$ & $5^{\circ} 03^{\prime} 58.23^{\prime \prime} \mathrm{E}$ & \multicolumn{2}{|c|}{ s.o. } & $x d m$ & $S \& A$ & Néant & 90 & 0,732 & 205 & 50,8 & 86 & 0 & & & & & \\
\hline 17 & $\mathrm{Br} 173$ & $50^{\circ} 29^{\prime} 50.06^{\prime \prime} \mathrm{N}$ & $5^{\circ} 01^{\prime} 48.94^{\prime \prime} \mathrm{E}$ & \multicolumn{2}{|c|}{ s.o. } & $x d m$ & Ca-Do & Illis. & 123 & 0,739 & 284 & 67,9 & 95,3 & 0 & 13,5 & & 13,5 & 26,9 & 64 \\
\hline 18 & $\mathrm{Na171a}$ & $50^{\circ} 29^{\prime} 08.78^{\prime \prime} \mathrm{N}$ & $4^{\circ} 59^{\prime} 54.34^{\prime \prime E}$ & 170,5 & 171 & $0,5 \mathrm{~m}$ & Ca-Do & 551 & 94 & 0,731 & 259 & 73,2 & 72 & 0 & 13,5 & & 19 & 26,9 & 91 \\
\hline 19 & $\mathrm{Na} 171 \mathrm{~b}$ & $50^{\circ} 29^{\prime} 08.78^{\prime \prime} \mathrm{N}$ & $4^{\circ} 59^{\prime} 54.34^{\prime \prime} \mathrm{E}$ & 170,5 & 171 & $0,5 \mathrm{~m}$ & Ca-Do & 551 & 120 & 0,762 & 436 & 79,3 & 95,3 & 0 & & & & & \\
\hline 20 & La168 & $50^{\circ} 30^{\prime} 47.96^{\prime \prime} \mathrm{N}$ & $5^{\circ} 04^{\prime} 28.52^{\prime \prime} \mathrm{E}$ & 166,5 & 168 & $1,5 \mathrm{~m}$ & $S \& A$ & Néant & 109 & 0,763 & 299 & 80 & 92,4 & 0 & 13,5 & & 13,5 & 19 & 64 \\
\hline 21 & Ch166a & $50^{\circ} 29^{\prime} 07.72^{\prime \prime} \mathrm{N}$ & $4^{\circ} 54^{\prime} 30.87^{\prime \prime} \mathrm{E}$ & \multicolumn{2}{|c|}{ s.o. } & $x \mathrm{dm}$ & Ca-Do & Néant & 122 & 0,735 & 209 & 66,5 & 92,5 & 1 & 13,5 & & 13,5 & 19 & 32 \\
\hline 22 & Ch166b & $50^{\circ} 29^{\prime} 07.72^{\prime \prime} \mathrm{N}$ & $4^{\circ} 54^{\prime} 30.87^{\prime \prime} \mathrm{E}$ & \multicolumn{2}{|c|}{ s.o. } & $x d m$ & Ca-Do & Illis. & 66 & 0,725 & 123 & 56,8 & 95,5 & 0 & & & & & \\
\hline 23 & Be140 & $50^{\circ} 28^{\prime} 47.94^{\prime \prime} \mathrm{N}$ & $4^{\circ} 55^{\prime} 22.77^{\prime \prime E}$ & 5. & o. & $x \mathrm{dm}$ & Ca-Do & 817 & 132 & 0,739 & 290 & 51,6 & 78 & 0 & 13,5 & & 13,5 & 26,9 & 64 \\
\hline & Moyenne & & & & & & & & 101 & 0,745 & 236 & 70 & 88 & 0 & 15,4 & 26,9 & 15,2 & 23 & 55,99 \\
\hline & $q 2 m$ & & & & & & & & & & & & & & & & & & \\
\hline 24 & Par179 & $50^{\circ} 31^{\prime} 10.36^{\prime \prime} \mathrm{N}$ & $5^{\circ} 08^{\prime} 45.15^{\prime \prime} \mathrm{E}$ & 175 & 179 & $4 \mathrm{~m}$ & S\&A & 89 & 72 & 0,693 & 188 & 51,7 & 62,2 & 0,5 & 26,9 & & 19 & 38 & 90 \\
\hline 25 & Par178.5 & $50^{\circ} 31^{\prime} 10.36^{\prime \prime} \mathrm{N}$ & $5^{\circ} 08^{\prime} 45.15^{\prime \prime} \mathrm{E}$ & 175 & 179 & $4 \mathrm{~m}$ & S\&A & 89 & 78 & 0,707 & 206 & 38,9 & 59,7 & 0 & & & & & \\
\hline 26 & Par178 & $50^{\circ} 31^{\prime} 10.36^{\prime \prime} \mathrm{N}$ & $5^{\circ} 08^{\prime} 45.15^{\prime \prime} \mathrm{E}$ & 175 & 179 & $4 \mathrm{~m}$ & $S \& A$ & 89 & 105 & 0,701 & 223 & 49,8 & 50,9 & 2,2 & & & & & \\
\hline 27 & Par177 & $50^{\circ} 31^{\prime} 09.56^{\prime \prime} \mathrm{N}$ & $5^{\circ} 08^{\prime} 45.72^{\prime \prime} \mathrm{E}$ & 175 & 179 & $4 \mathrm{~m}$ & $S \& A$ & 89 & 89 & 0,694 & 335 & 36,6 & 45,5 & 0 & & & & & \\
\hline 28 & Par 176,5 & $50^{\circ} 31^{\prime} 09.56^{\prime \prime} \mathrm{N}$ & $5^{\circ} 08^{\prime} 45.72^{\prime \prime} \mathrm{E}$ & 175 & 179 & $4 \mathrm{~m}$ & $S \& A$ & 89 & 97 & 0,706 & 255 & 40,2 & 64,6 & 0 & & & & & \\
\hline 29 & Par176 & $50^{\circ} 31^{\prime} 09.56^{\prime \prime} \mathrm{N}$ & $5^{\circ} 08^{\prime} 45.72^{\prime \prime} \mathrm{E}$ & 175 & 179 & $4 \mathrm{~m}$ & $S \& A$ & 89 & 109 & 0,719 & 310 & 37,9 & 42,3 & 0,3 & & & & & \\
\hline 30 & Sc174a & $50^{\circ} 29^{\prime} 06.11^{\prime \prime} \mathrm{N}$ & $5^{\circ} 02^{\prime} 44.48^{\prime \prime} \mathrm{E}$ & Inc. & 175 & Inc. & $S \& A$ & 561 & 98 & 0,696 & 282 & 42 & 29,1 & 0 & 19 & & 19 & 26,9 & 64 \\
\hline 31 & Sc174b & $50^{\circ} 29^{\prime} 06.11^{\prime \prime} \mathrm{N}$ & $5^{\circ} 02^{\prime} 44.48^{\prime \prime} \mathrm{E}$ & Inc. & 175 & Inc. & $S \& A$ & 561 & 95 & 0,73 & 242 & 41,3 & 37,2 & 0,4 & & & & & \\
\hline 32 & Lo165a & $50^{\circ} 28^{\prime} 02.02^{\prime \prime} \mathrm{N}$ & $4^{\circ} 56^{\prime} 32.54^{\prime \prime E}$ & Inc. & 165 & Inc. & Ca-Do & Illis. & 92 & 0,709 & 331 & 32,5 & 71,6 & 5,4 & 26,9 & & 26,9 & 38 & 91 \\
\hline 33 & Lo165b & $50^{\circ} 28^{\prime} 02.02^{\prime \prime} \mathrm{N}$ & $4^{\circ} 56^{\prime} 32.54^{\prime \prime} \mathrm{E}$ & Inc. & 165 & Inc. & Ca-Do & Illis. & 45 & 0,695 & 207 & 24,1 & 60 & 6,8 & & & & & \\
\hline & Moyenne & & & & & & & & 88 & 0,705 & 258 & 39,5 & 52,3 & 1,56 & 24,3 & & 21,6 & 34,3 & 81,7 \\
\hline 34 & Lv137 & $50^{\circ} 27^{\prime} 38.38^{\prime \prime} \mathrm{N}$ & $4^{\circ} 55^{\prime} 25.04^{\prime \prime} \mathrm{E}$ & 135 & 140 & 5 & S\&A & Illis. & 65 & 0,717 & 205 & 32,4 & 40,9 & 0 & 13,5 & 38 & 19 & 38 & 64 \\
\hline 35 & Er110 & $50^{\circ} 27^{\prime} 36.58^{\prime \prime} \mathrm{N}$ & $4^{\circ} 53^{\prime} 39.26^{\prime \prime E}$ & 110 & 115 & 5 & S\&A & Illis. & 121 & 0,688 & 220 & 22,7 & 64 & 0 & 13,5 & 26,9 & 13,5 & 19 & 64 \\
\hline 36 & An71 & $50^{\circ} 29^{\prime} 42.80^{\prime \prime} \mathrm{N}$ & $5^{\circ} 06^{\prime} 28.19^{\prime \prime} \mathrm{E}$ & 63 & 73 & 10 & $S \& A$ & Aucun & 103 & 0,71 & 998 & 16,3 & 23,8 & 0,7 & 26,9 & & 19 & 38 & 91 \\
\hline & Moyenne & & & & & & & & 88 & 0,706 & 343 & 31,4 & 47,4 & 1,9 & 20,7 & 32,5 & 19,8 & 32,4 & 75,94 \\
\hline
\end{tabular}

Tableau 1. Données de terrain et valeurs des différents indices sédimentologiques définis dans le texte. Explications : Parmi les niveaux q2m, le plus élevé de chaque transect (Pa175-179, Sc175 et Lo165) a été intégré dans un même groupe, ceux des niveaux inférieurs sont dans un groupe séparé. Échantillons : $\mathrm{n}^{\circ}=$ ordre de l'échantillon dans les Figures 15 à 18 ; Xy000= les lettres désignent le site localisé sur la Figure 1, et le nombre correspond à l'altitude à laquelle l'échantillon a été prélevé, les lettres a et b distinguent des échantillons qui ont été prélevés dans une même excavation. Données de terrain : $\mathrm{LbX}=$ latitude du point de prélèvement en coordonnées Lambert ; $\mathrm{LbY}=$ longitude du point de prélèvement en coordonnées Lambert ; Base= altitude de la base du cailloutis ; Toit= altitude du toit du cailloutis ; s.o.= sans objet pour des cailloutis d'épaisseur décimétrique ; Épais= épaisseur du cailloutis, dont x dcm (=épaisseur d'ordre décimétrique) ; Socle : S\&A= roches siliceuses et/ou argileuses (grès, schistes,...) $; \mathrm{Ca}-\mathrm{Do}=$ roches solubles (calcaire, dolomie,...); $\mathrm{N}^{\circ}$ ds Clair.= numéro du replat dans Clairbois (1957). Indices de nature et de forme : $\mathrm{n}=$ nombre d'éléments ; $\mathrm{Iw}=$ indice d'arrondi de Wadell ; $\mathrm{Q}$ '= fréquence des quartz vs les autres cailloux émoussés (classe $8-16 \mathrm{~mm}$ ); Q" = fréquence des quartz vs quartzite (classe 8-16 mm); G'= fréquence des galets de quartz (émoussé marin) vs quartz à émoussé sommaire (classe 8-16 mm); Ca\%= fréquence des cailloux de calcaire et de dolomie. Indices granulométriques : Mo1= premier mode ; Mo2= deuxième mode ; Mé= médiane ; Q3= troisième quartile ; ‘+gros’= maille du premier tamis vide. 
gréso-schisteux, mais la dénivelée par rapport à leur site d'origine n'est jamais supérieure au décamètre ; on peut donc supposer une relation de provenance avec le replat surplombant.

\section{A. Indice de fréquence des quartz}

En se basant uniquement sur l'émoussé, la formule écarte volontairement la prétention de faire des distinctions entre les diverses roches paléozoïques de l'Ardenne s.l. En fait, on accepte entre autres que la décroissance de cet indice traduise l'incision des cours d'eau dans les altérites des roches paléozoïques (où le quartz a mieux résisté à l'altération que la roche hôte), puis dans les roches non altérées. Les résultats sont représentés dans la Figure 7.

Indépendamment des réserves formulées plus haut sur l'altitude des échantillons, on constate une tendance à la diminution de l'indice de fréquence des quartz en fonction de la diminution de l'altitude. Les Onx de plateau et ceux des vastes replats de Brichebo (Br187) et de Landenne (La183) ont les indices les plus élevés; le replat du zoning de Landenne (La168) est dans le même cas bien que se trouvant à une altitude nettement inférieure, mais il est dans un site de paléo-confluence entre deux ruisseaux issus du plateau de Petit-Waret. Des apports juvéniles moins riches en quartz s'ajoutent dans des graviers sous la zone de superposition avec les $O n x$ de plateau. Il n'y a pratiquement pas de superposition entre les champs d'Onx et de $q 2 m$. Dans ces derniers, en général l'indice décroît avec l'altitude. On remarquera aussi qu'à altitude identique, les échantillons du flanc droit ont des indices plus faibles que ceux du flanc gauche. On notera également la variabilité systématique pour 2 échantillons d'un même site.

\section{B. Indice d'arrondi de Wadell (Iw)}

Il est bien connu que l'émoussé et le polissage des éléments évoluent beaucoup plus rapidement sur les plages lorsqu'ils sont déplacés en permanence par la houle. Par contre, dans les cours d'eau, l'usure ne s'exerce que si les cailloux se déplacent ou sont en affleurement sur le lit mineur où ils sont soumis au sablage par les charges en saltation et en suspension. Dans le cas présent, cet indice $I w$ permet de faire la distinction entre les deux populations de quartz précitées. On notera également

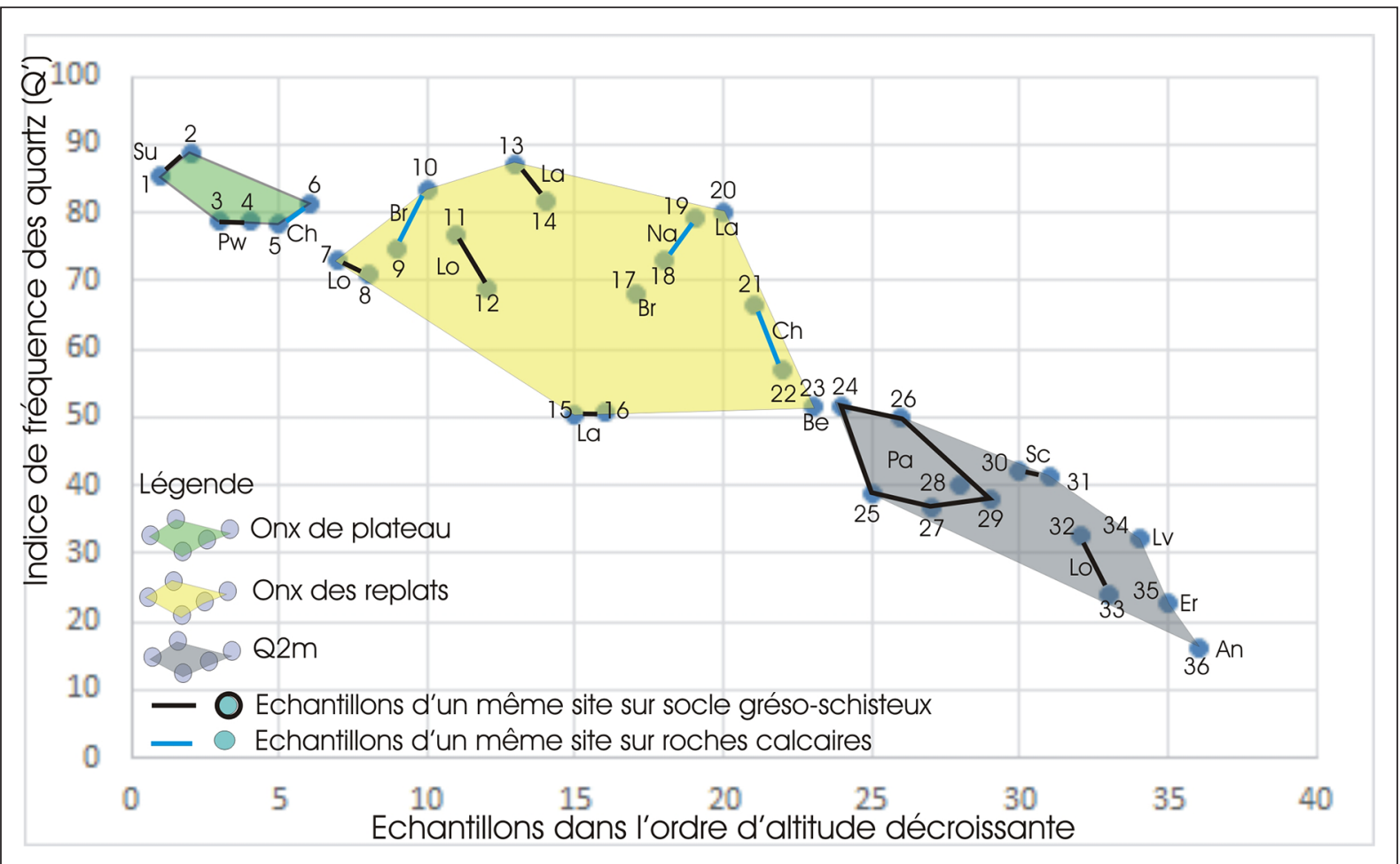

Figure 7. Indice de fréquence des quartz purs par rapport à l'ensemble des cailloux émoussés d'autres natures. Les labels des sites sont ceux du tableau 1 . Formule spécifique : Q' $=100 \times \mathrm{Q} /(\mathrm{Q}+\mathrm{Nq})$ dans laquelle $\mathrm{Q}=$ nombre de quartz purs émoussés, entiers ou cassés ; $\mathrm{Nq}=$ nombre des éléments émoussés entiers ou cassés de nature autre que le quartz pur). N.B. Un élément qui contient du quartz sous forme de veine dans du quartzite hôte n'est pas un quartz pur. 

entre Namur et Huy (Belgique) à la suite de la capture de la Semois

la variabilité systématique pour 2 échantillons d'un même site. Les résultats sont représentés à la Figure 8.

Indépendamment des réserves formulées sur l'altitude des échantillons, on constate une tendance à la diminution de l'indice d'arrondi de Wadell en fonction de la diminution de l'altitude, ce qui implique un accroissement de la fréquence des quartz à émoussé sommaire, soit essentiellement fluviatile. Les sites de plus basse altitude sur le flanc gauche de la vallée (La175, La177, Br173, Ch166 et Be140) échappent à la superposition avec les valeurs des sites de plateau, ce qui atteste un apport juvénile d'éléments moins bien arrondis. Sur le flanc droit de la vallée, l'indice Iw est nettement décroissant en fonction de l'altitude de Lo198 ( 0.76), Lo184( $\sim 0.74$ ) puis Lo165( 0.70). Il n'y a pratiquement pas de superposition entre les champs d'Onx et de $q 2 m$. Dans ces derniers, il n'y a pas de décroissance de Iw avec la diminution d'altitude sur le flanc gauche. On notera également la variabilité systématique pour 2 échantillons d'un même site.

\section{Indice de polissage des quartz}

La qualité discriminante de cet indice relève des mêmes conditions d'usure que celle évoquées pour l'indice $I w$. Les résultats sont représentés à la Figure 9.

Indépendamment des réserves formulées plus haut sur l'altitude des échantillons, on constate que le faciès des $O n x$ de plateau et des replats est pour l'essentiel identique. Par contre, il n'y a pas de superposition des échantillons $q 2 m$ avec les Onx quels qu'ils soient. On notera également la variabilité pour 2 échantillons d'un même site.

\section{Indices granulométriques}

Toutes les courbes granulométriques originales sont groupées dans des planches en annexe dans Juvigné et al. (2020a). Les indices particulièrement discriminants sont repris dans le Tableau 1. Ici la lecture des résultats ne porte que sur les moyennes par groupe dans la mesure où les lentilles d'un même dépôt fluviatile ont elles-mêmes des textures différentes. Il en ressort qu'il existe une gradation granulométrique : (1) les $O n x$ de plateau sont particulièrement fins (ex. : $\mathrm{D}_{50}=9,9 \mathrm{~mm}$ ); (2) des éléments plus grossiers s'ajoutent aux précédents pour constituer les $O n x$ des replats (ex. : $\mathrm{D}_{50}=15,2$; (3) des éléments très nettement plus grossiers s'ajoutent aux précédents, que l'on considère le seul gravier de Paradis (ex. : $\mathrm{D}_{50}=19 \mathrm{~mm}$ ) ou le gravier

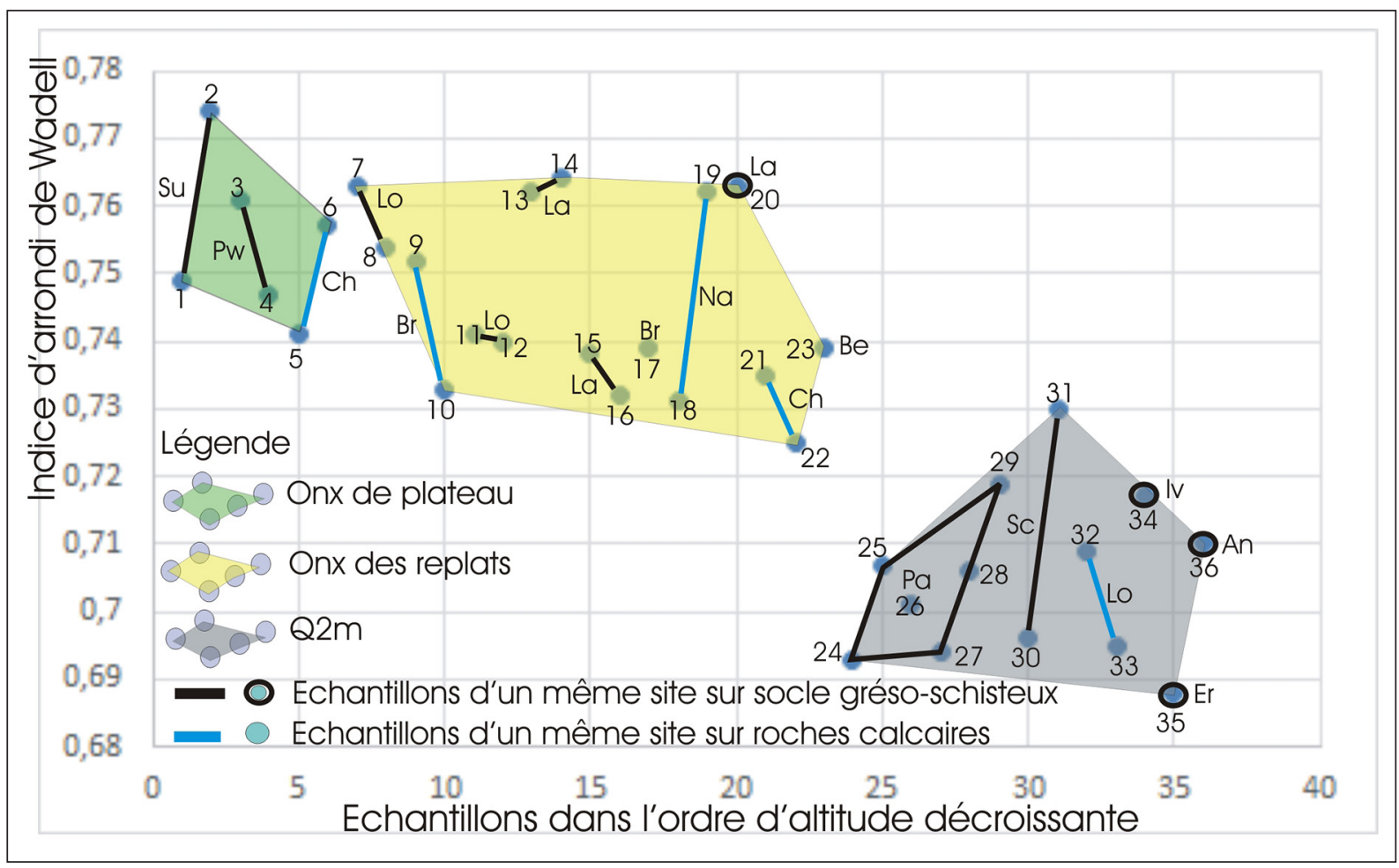

Figure 8. Indice d'arrondi de Wadell pour les quartz purs (entiers et cassés). Explication : les labels des sites sont ceux du Tableau 1. 


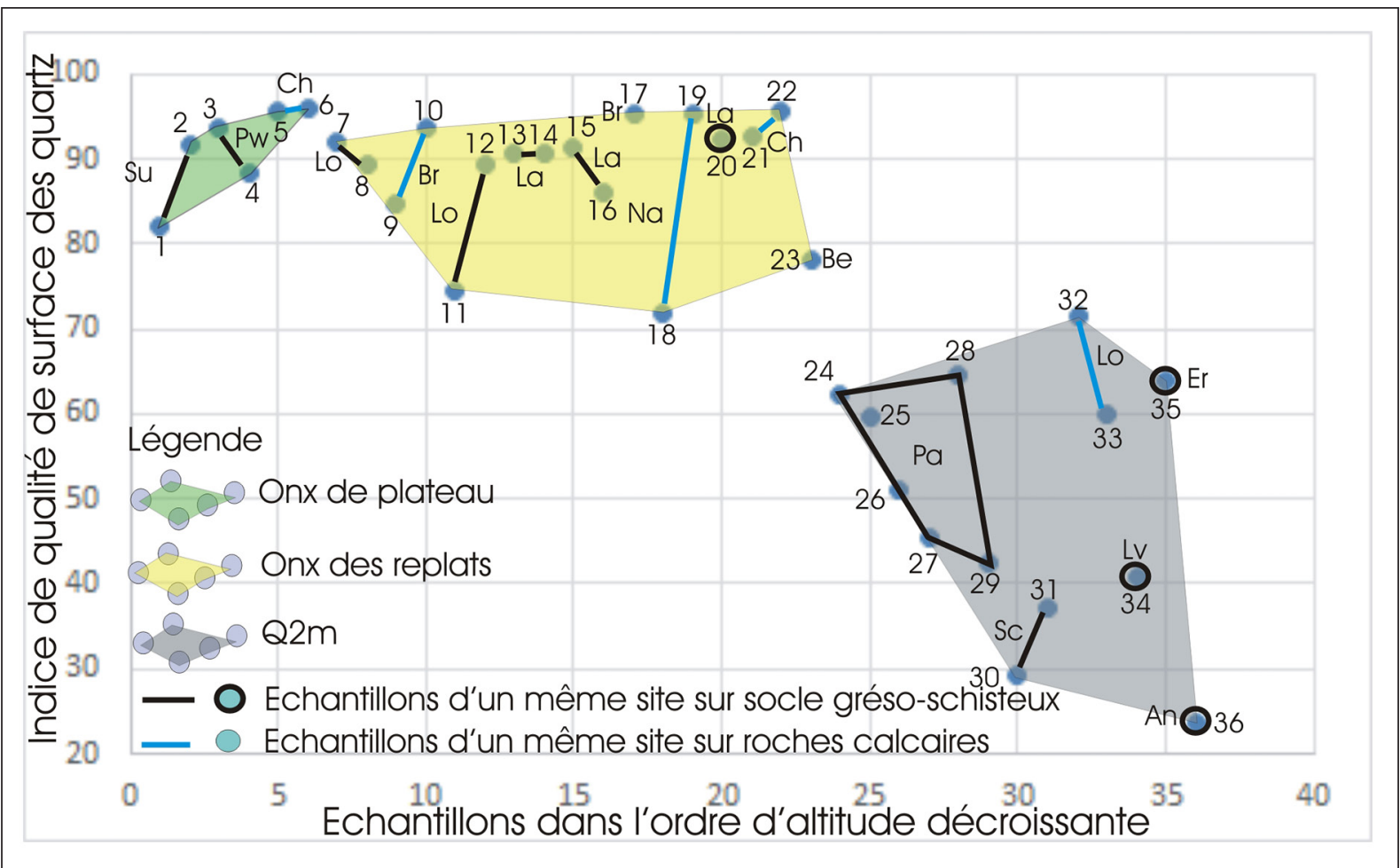

Figure 9. Indice de polissage des quartz. Explications : les labels des sites sont ceux du tableau 1 ; Formule : $\mathrm{G}^{\prime}=$ $100 * \mathrm{Gq} /(\mathrm{Gq}+\mathrm{Cq})$ avec $\mathrm{Gq}=$ nombre de galets de quartz entiers ou cassés qui ont conservé une partie de surface bien émoussée et bien polie ; $\mathrm{Cq}=$ nombre de cailloux roulés de quartz à émoussé sommaire c'est-à-dire qui n'ont aucune partie bien émoussée et bien polie.

$q 2 m$ le plus élevé de chaque tronçon revisité (ex. : $\left.\mathrm{D}_{50}=21,6 \mathrm{~mm}\right)$.

\section{E. Les cailloux de plus de $16 \mathrm{~mm}$ dans les cail- loutis q2m}

Dans les cailloutis Onx, les cailloux de plus de $16 \mathrm{~mm}$ sont peu fréquents (voir annexe) et une étude statistique nécessiterait des quantités d'échantillons irrationnelles, mais dans le lambeau $q 2 \mathrm{~m}$ de Paradis, ils sont très abondants et l'association est la suivante pour $n=450$ : quartz à émoussé sommaire $22,9 \%$; galets de quartz $11,1 \%$; quartzite avec veine de quartz et/ou empreinte de pyrite $19,8 \%$; cailloux arrondis à surface lisse (quartzite ?) 7,1\% ; cailloux plats et lisses (quartzo-phyllade et/ou phyllade ?) 16,2 $\%$; conglomérats à matrice $\mathrm{Fe}-\mathrm{Mn} 2 \%$; grès gris clair $2,9 \%$; divers cailloux émoussés $17,1 \%$ et divers cailloux anguleux $8 \%$. Aucun carbonate n'a été trouvé. Sur le plan qualitatif l'association est semblable à celle de la classe de 8 à $16 \mathrm{~mm}$, et sur le plan quantitatif il est bien connu que les fréquences changent d'une classe granulométrique à l'autre. Le présent résultat fait office de référence pour des comparaisons ultérieures.

\section{F. Synthèse des caractéristiques des graviers}

Les différences essentielles entre les trois groupes de graviers distingués ci-dessus sont représentées dans la Figure 10.

Le contraste le plus remarquable entre les deux cailloutis est illustré par la différence relative à la nature et à la forme des cailloux : (1) les galets de quartz constituent l'essentiel du gravier depuis le niveau du plateau de Hesbaye jusqu'à la terrasse de Landenne à $183 \mathrm{~m}$; quelques quartzites viennent en deuxième lieu tandis que l'ensemble des autres galets (grès, lydite, phtanite, kieseloolithes...) se comptent ensemble en quelques pourcents. Au cours de l'incision, la granularité du gravier Onx a aussi légèrement augmenté. L'émoussé des éléments a de toute évidence été acquis sur des plages marines.

Le gravier de Paradis/Couthuin est nettement plus grossier et dominé par des grès divers et des quartzites plus fréquents. L'émoussé des quartz est bimodal avec des éléments repris à la dernière terrasse Onx, et d'autres dont l'émoussé sommaire atteste qu'ils n'ont subi l'usure qu'en milieu fluviatile. Il faut ajouter que l'épaisseur du cailloutis passe 


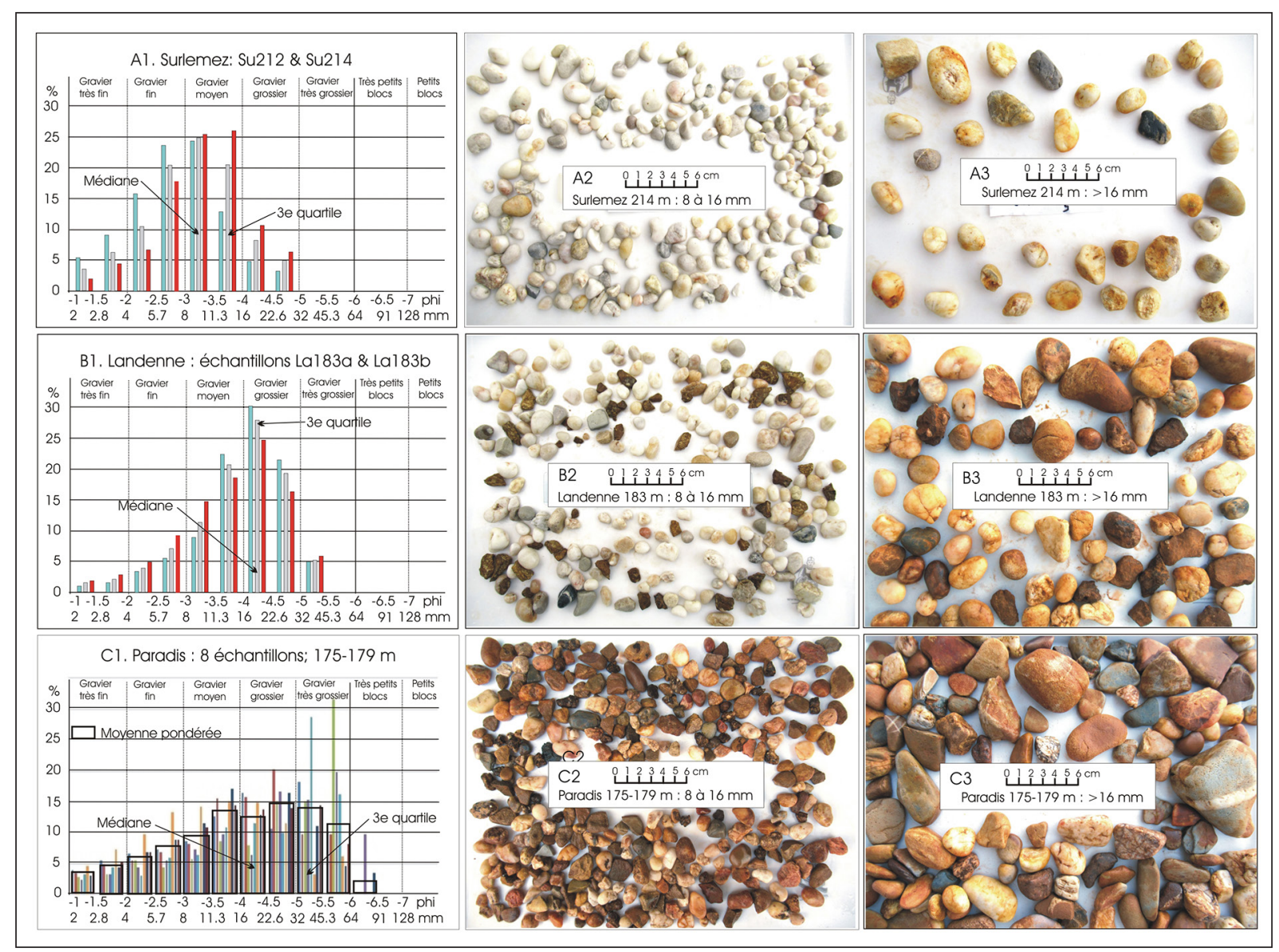

Figure 10. Le gravier Onx depuis le plateau de Hesbaye namuroise à 213-217 m (A), jusqu'au replat de Landenne à $183 \mathrm{~m}(\mathrm{~B})$, et le cailloutis q2m de Paradis/Couthuin à 175-179 m (C). A1, A2, A3= distribution granulométrique du gravier ; B1, B2, B3= gravier moyen $(8$ à $16 \mathrm{~mm}) ; \mathrm{C} 1, \mathrm{C} 2, \mathrm{C} 3=$ gravier grossier et très grossier $(>16 \mathrm{~mm})$. Explications : en A1 et B1, la colonne grise représente la moyenne pondérée des distributions des deux échantillons ; en $\mathrm{C} 1$, c'est le rectangle noir qui représente la moyenne pondérée de 8 échantillons.

brutalement de l'ordre pluri-décimétrique (Onx des replats) à 4 m d'épaisseur à Paradis.

\section{G. Minéraux denses transparents (Mdt)}

Quelques échantillons représentatifs des différents niveaux ont été retenus pour les déterminations. Les résultats sont rassemblés dans le Tableau 2.

La part des mdt déterminables varie de 1,5 à $39,2 \%$; la majorité des grains dits indéterminables consistent en minéraux opaques et en débris rocheux divers qui ne diffusent pas les propriétés optiques nécessaires à la détermination. Parmi les minéraux limpides déterminables, moins de $3 \%$ n'ont pas pu être déterminés avec une conviction suffisante. Les minéraux des sables $O n x$ sont en général un peu plus petits ( $89 \%$ de $75-150 \mu \mathrm{m})$ que ceux des $q 2 \mathrm{~m}$ ( $85 \%$ de $75-150 \mu \mathrm{m})$; tous les autres minéraux sont dans la classe de 150 à $300 \mu \mathrm{m}$ ), donc aucun grain de 350 à $425 \mu \mathrm{m}$ n'a été récolté. Dans tous les cas, les ubiquistes (zircon, tourmaline et polymorphes $\mathrm{du} \mathrm{TiO}_{2}$ ) constituent le groupe dominant avec 67 à $88 \%$, suivi par le groupe des minéraux appelés communément paramétamorphiques (staurotide, disthène, andalousite, topaze et sillimanite) avec 4,5 à $30,6 \%$. Les Onx comptent en moyenne davantage d'ubiquistes que les $q 2 m$ de Paradis : $82,3 \%$ vs $76,9 \%$, mais le rapport est inverse pour les paramétamorphiques (13,8 vs 19,5\%). Les parts des autres minéraux sont toujours faibles y compris dans le lbt de Paradis/Couthuin dans lequel sept échantillons répartis stratigraphiquement dans le cailloutis ont été traités. En fait, ces spectres sont le reflet de ceux des sables marins oligocènes (Bourguignon, 1954; Demoulin, 1987). Toutefois, dans les terrasses $q 2 m$ du flanc droit de la vallée (Sclayn), la part des paramétamorphiques est nettement plus faible qu'à Paradis (flanc gauche). Il faut sans doute voir là le résultat d'une influence locale; il n'y a pas de vaste 


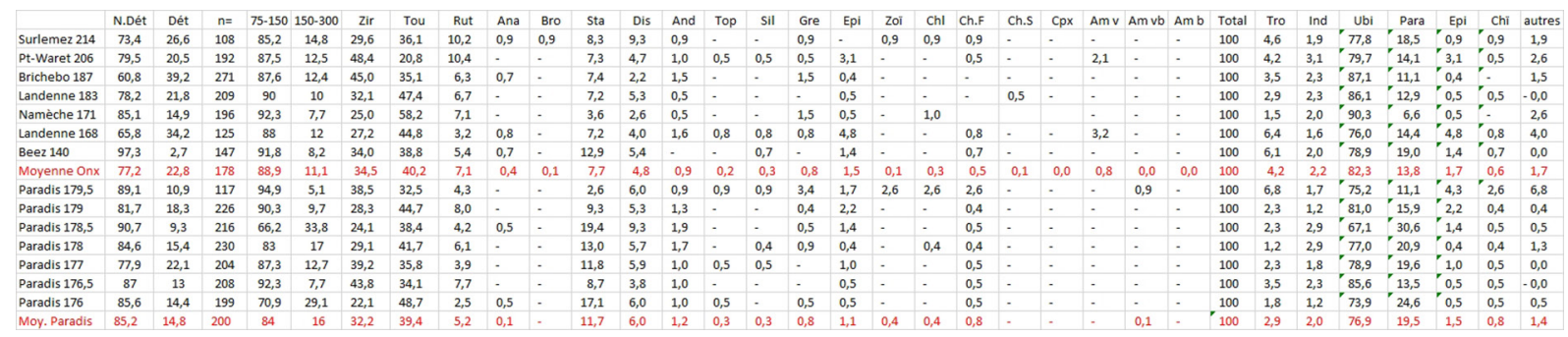

Tableau 2. Association de minéraux denses transparents dans des terrasses de la Meuse entre Namur et Huy. Légende : N.dét. $=$ non déterminables (en $\%$ du total des grains) ; Dét.= déterminables (en \% du total des grains) $; n=$ nombre de mdt déterminés ; 75-150 et 150-300= classes granulométriques ; Zir=zircon ; Tou= tourmaline ; Rut= rutile ; $\mathrm{Ana}=$ anatase $; \mathrm{Bro}=$ brookite $; \mathrm{Sta}=$ staurotide $; \mathrm{Dis}=$ disthène $; \mathrm{And}=$ andalousite $; \mathrm{Top}=$ topaze $;$ Sil $=$ sillimanite ; Gre= grenat ; Epi= épidote $;$ Zoï= zoïsite $; \mathrm{Chl}=$ chlorite $; \mathrm{Ch} . \mathrm{F}=$ chloritoïde en feuillets ; Ch.S $=$ chloritoïde du Serpont $; \mathrm{Cpx}=$ clinopyroxène $; \mathrm{Am} . \mathrm{v}=$ amphibole verte $; \mathrm{Am} . \mathrm{vb}=$ amphibole vert-brun ; $\mathrm{Am} . \mathrm{b}=$ amphibole brune ; Tro= trouble indéterminé ; Ind.= limpide indéterminé.

nappe de sable marin oligocène sur le flanc droit de la vallée, tandis que Paradis se trouve au pied de la colline de Surlemez qui en porte encore une nappe épaisse. On notera aussi la présence de deux types de chloritoïdes dont les roches mères sont dans les massifs cambro-ordoviciens de Haute Belgique : (1) le chloritoïde en feuillets qui est le plus largement répandu ; (2) des cristaux massifs et limpides du Serpont (Juvigné et al., 2020b). Les occurrences de ces minéraux sont irrégulières dans les cailloutis $O n x$, mais quasi systématiques dans les $q 2 m$.

Compte tenu de la postériorité de la capture de la Meuse lorraine par rapport à celle de la Semois (Dolfus, 1900 ; Pissart, 1961), une recherche particulière a été réalisée pour s'assurer qu'il existe bien une terrasse inférieure à celle de Paradis/Couthuin (Cou-Par[b175 ; s179 ; d100] dans laquelle les amphiboles des Vosges (Bustamante, 1973) entrent en scène. L'arrivée des premières amphiboles a été établie dans deux lbxt dont les cailloutis se situent entre 110 et $120 \mathrm{~m}$, soit $+80 \mathrm{~m}$ vs plaine d'inondations (Tableau 1) : Fluxis 110 (Chemin d'Erpent/ Namur Est) et Jambes 117 (Jambes cimetière) (revoir la Figure 1). Cette observation a initié une étude particulière dans la mesure où il existe un consensus dans la littérature selon lequel la capture de la Meuse lorraine par la Meuse de Dinant aurait eu lieu pendant la transition mio-pliocène (Pissart et al., 1997a) (Juvigné et Houbrechts, ce fascicule).

\section{COUPES TRANSVERSALES SCHÉ- MATIQUES ET PROFIL LONGITUDINAL ENTRE NAMUR ET HUY}

Les coupes transversales schématiques des différents tronçons renseignent sur les étapes de l'évolution de la partie supérieure de la vallée.

\section{A. Le tronçon d'Andenne à Gives}

Il s'agit ici d'une figure de référence dans la mesure où les cailloutis $O n x$ et $q 2 m$ sont les plus élevés de leurs groupes respectifs, et qu'ils reposent sur des terrains qui excluent l'effondrement par karstification (Figure 11). Dans ce tronçon, il n'y a aucun lbxt sur le flanc droit de la vallée, et à partir de ce site, la Meuse est restée sur le flanc gauche jusqu'à Huy où elle a notamment développé le méandre de Leumont. Celui-ci ne se recoupera qu'au niveau de la plus basse terrasse mosane (Juvigné et al., 2015).

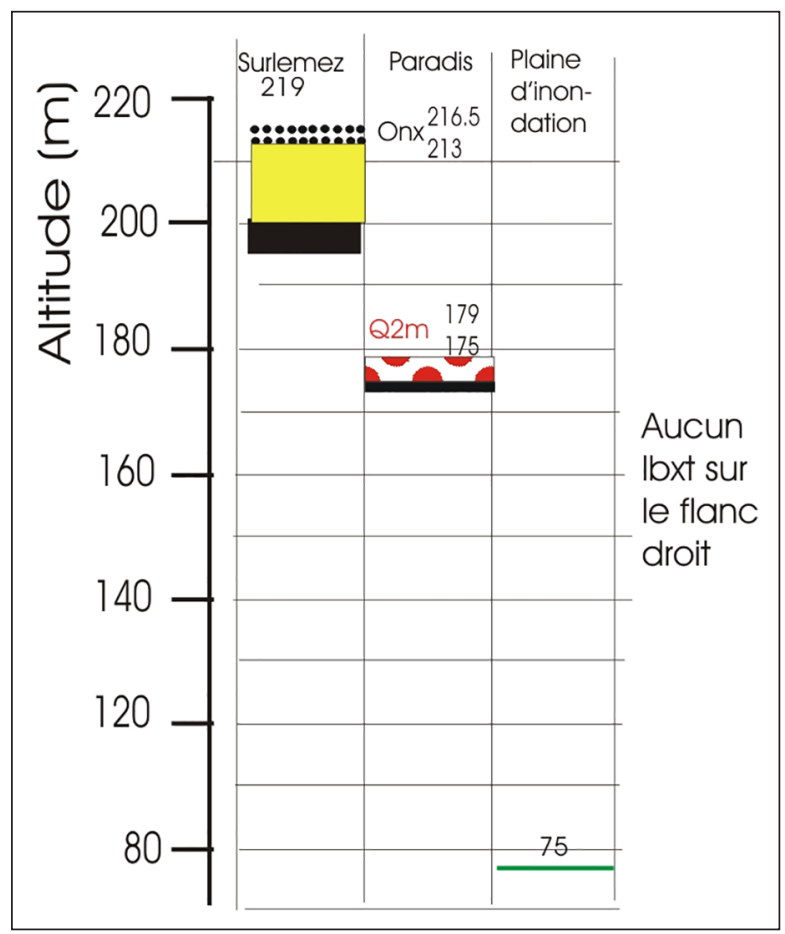

Figure 11. Coupe transversale schématique de lbxt de référence entre Andenne et Huy . Explications : pastilles noires $=$ cailloutis Onx ; rectangle jaune $=$ dépôts marins oligocènes ; plage noire $=$ socle gréso-schisteux (Houiller) ; pastilles rouges $=$ cailloutis $\mathrm{q} 2 \mathrm{~m}$. 

entre Namur et Huy (Belgique) à la suite de la capture de la Semois

\section{B. Le tronçon de Namèche à Andenne}

Sur la retombée méridionale du plateau de Petit-Waret, il existe du cailloutis Onx sur trois replats : (1) Chapelle St Mort (190-191 m) sur socle gréso-schisteux ; (2) Brichebo (185-186,5 m) sur socle dolomitique ; (3) Landenne (183-185 m) sur socle gréso-schisteux. On notera que la base du lbt Onx de Landenne ( 183 m) n'est que $\sim 3 \mathrm{~m}$ plus haut que le toit du cailloutis $q 2 m$ de Paradis (179 m) (Figure 12).

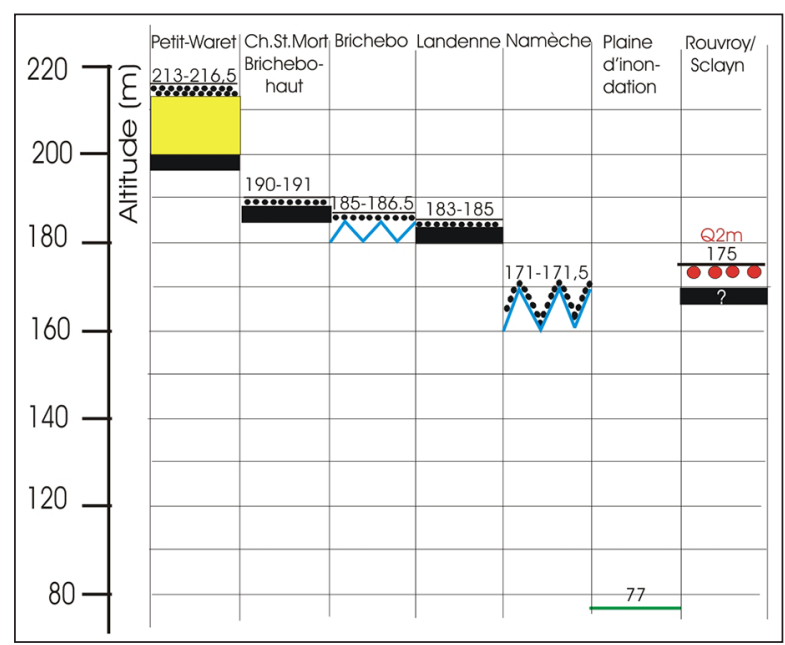

Figure 12. Coupe transversale schématique du tronçon Namèche-Andenne. Explications : pastilles noires= cailloutis Onx ; rectangle jaune $=$ dépôts oligocènes ; plage noire $=$ socle gréso-schisteux (Houiller) ; pastilles rouges $=$ cailloutis $\mathrm{q} 2 \mathrm{~m}$; trait bleu= socle calcaire ou dolomitique ; point d'interrogation= base inconnue.

On notera que le cailloutis $O n x$ de la carrière de dolomie (Lhoist) tapisse une surface karstifiée sous le niveau du lambeau de Rouvroy/Sclayn (toit du $q 2 \mathrm{~m}$ à $175 \mathrm{~m}$ ) sur le flanc opposé. Il ne peut représenter une terrasse Onx que si on admet que le cailloutis s'est mis en place plus haut que le toit du cailloutis $q 2 m$ de Rouvroy/Sclayn, ce qui implique un effondrement post sédimentaire par karstification d'au moins $8 \mathrm{~m}$. Comme il est avéré que dans ce tronçon la Meuse se situait sur le flanc droit de sa vallée actuelle, il devrait s'agir d'une surface d'érosion à $171 \mathrm{~m}$ qui se raccordait à une terrasse mosane inférieure du flanc droit de la vallée.

\section{Le tronçon de Namur à Namèche}

La coupe transversale de la Figure 13 rassemble non seulement nos observations, mais aussi des données provenant des archives du Service géologique.

La morphologie en gradins des lbxt Onx est particulièrement développée sur le flanc gauche entre
Champion et Beez, où il n'y a que des cailloutis Onx identifiés depuis le plateau jusqu'au replat portant les échantillons Ch166a\&b. Sur le flanc droit, le cailloutis Onx le plus élevé est à Loyers-Taillette (LoyTai[b198; s199; d119]), mais il est néanmoins $10 \mathrm{~m}$ plus bas que le lbt du plateau de Champion (208 m). Quant au lbt $q 2 m$ le plus élevé, il est à Loyers sur le flanc droit (Loy-StJ[b160; s165; d81]). Sur le flanc gauche à Beez à $150 \mathrm{~m}$ d'altitude Stainier (1894) et Clairbois (1957) mentionnent la présence de lbxt $q 2 m$. La dénivelée entre le toit du lbt $q 2 m$ le plus élevé (165 m) et la base du plus bas lbt Onx ( 168 m).

\section{Profil longitudinal de la limite Onx vs q2m entre Namur et Huy}

On dispose de trois sites pour tracer la limite précitée (Figure 14) : (1) dans le tronçon de référence de Landenne à Paradis sur socle siliceux, la limite se trouve entre $183 \mathrm{~m}$ et $179 \mathrm{~m}$; (2) dans la coupe de Champion-Loyers sur calcaires et dolomies, elle se trouve entre 168 et $165 \mathrm{~m}$; (3) sur le lbt de Rouvroy-Sclayn, le toit du cailloutis $q 2 m$ sur socle houiller est à $175 \mathrm{~m}$. De ce fait, la limite est en contre-pente depuis Namur jusqu'à Huy. En combinant la coupe transversale de Champion-Loyers (Figure 13) et la coupe longitudinale (Figure 14), on constate que la région de Namur pourrait être à grande échelle une dépression karstique dans laquelle ni les sables marins, ni les lbxt n'auraient conservé leur altitude originelle. En conclusion, l'altitude du changement pétrographique n'est fiable que sur socle gréso-schisteux, soit en ne tenant compte que des sites de Petit-Waret, Landenne, Surlemez, Paradis, où il se trouve entre $183 \mathrm{~m}$ et $179 \mathrm{~m}$.

\section{V. ÉVOLUTION DE LA VALLÉE DE LA MEUSE DE NAMUR JUSQU'À HUY}

\section{A. Le dépôt Onx du Mont Saint-Héribert (Namur-Sud)}

Il s'agit du cailloutis Onx le plus élevé connu dans la région namuroise ; il se trouve à $\sim 235 \mathrm{~m}$ d'altitude, soit une vingtaine de mètres plus haut que les nappes de Petit-Waret et de Surlemez (Figure 15). Sa présence en plusieurs mètres d'épaisseur a été démontrée dans une série de puits dans le cadre de la préparation de la construction du fort (Van den Broeck et Rutot, 1888b). Il est inaccessible et n'a donc pu être échantillonné. C'est de toute évidence 


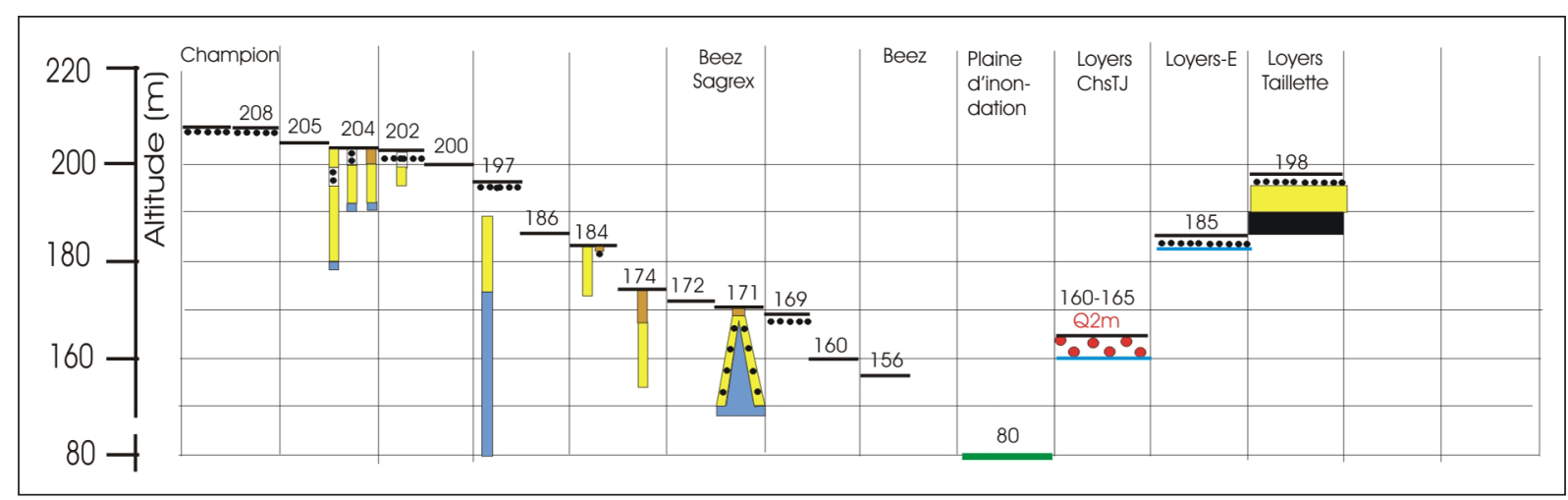

Figure 13. Coupe transversale schématique du tronçon Champion-Loyers. Explications : trait noir= replat ; pastilles noires $=$ cailloutis Onx $;$ colonne ocre $=$ loess $;$ colonne jaune $=$ dépôts marins oligocènes $;$ plage noire $=$ socle gréso-schisteux (Houiller) ; pastilles rouges $=$ cailloutis $\mathrm{q} 2 \mathrm{~m}$; sigles bleus= socle de roches calcaires.

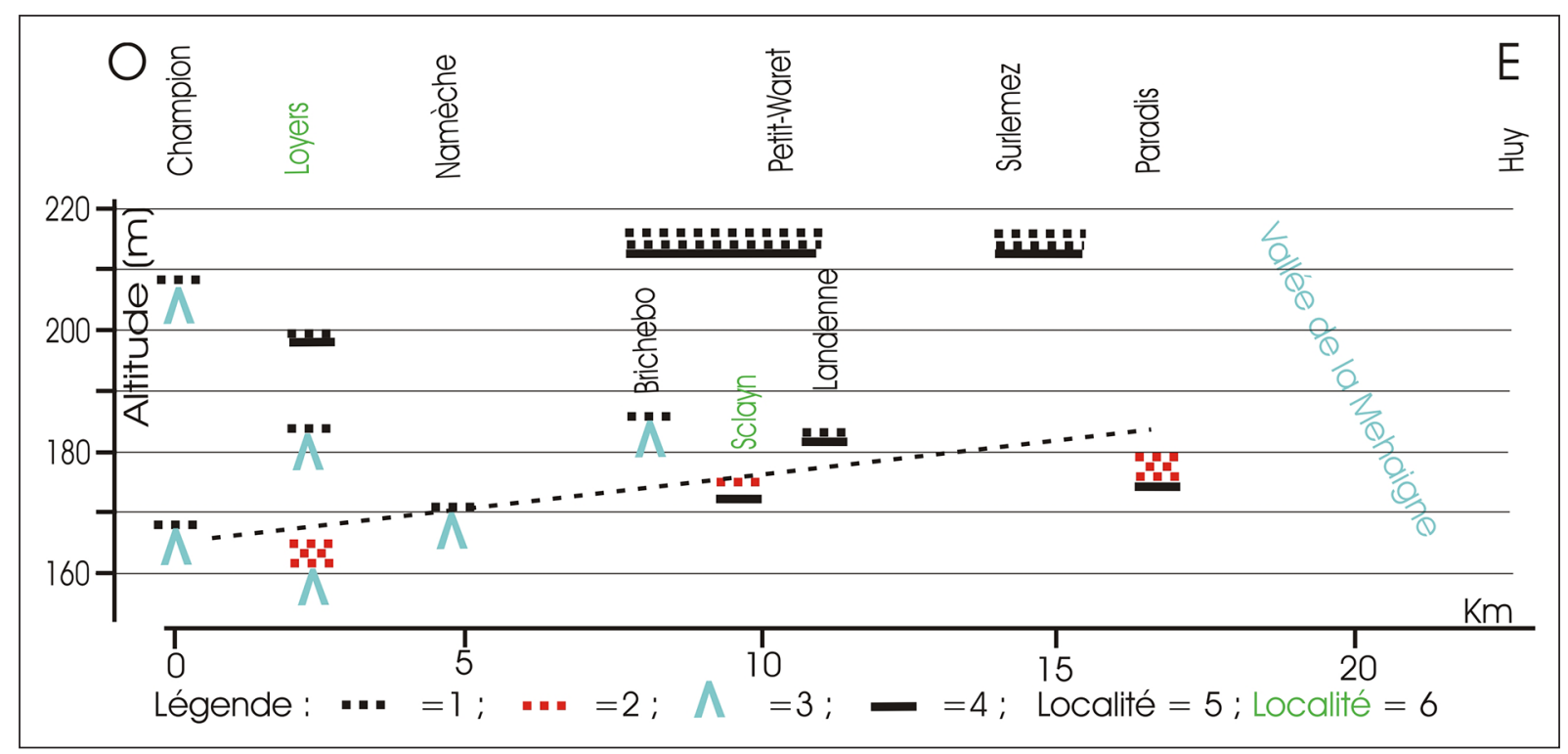

Figure 14. Profil longitudinal du changement sédimentologique depuis Loyers/Namur jusqu'à Paradis/Couthuin. Légende : $1=$ cailloutis Onx ; $2=$ cailloutis $\mathrm{q} 2 \mathrm{~m} ; 3=$ socle de roches calcaires $; 4=$ socle de roches grès et schistes ; $5=$ localités du flanc gauche $; 6=$ localités du flanc droit.

la trace la plus ancienne connue de la Meuse de Dinant primitive.

\section{B. Les dépôts Onx de plateau et la Meuse de Dinant}

Les cailloutis Onx des plateaux de Surlemez (b213,5 ; s216,5) et de Petit-Waret (b213 ; s216,5) sont à une altitude identique, et une vingtaine de mètres plus bas que le dépôt du Mont Saint-Héribert (Figure 16). Sous tous ces dépôts, il n'existe pas de roches susceptibles d'avoir provoqué des effondrements karstiques. À l'un et l'autre niveau, le prolongement de l'écoulement qui a déposé les cailloutis est indéterminable au-delà de la crête de Hesbaye namuroise, et l'avoir attribué à une Meuse primitive sur la base du seul parallélisme avec le cours d'eau actuel (Stainier ; 1894 ; Macar, 1945) est peu fondé. Tout ce que l'on peut en dire, c'est qu'il doit avoir été mis en place par la Meuse de Dinant (Figure 15), et que le faciès de plage marine du gravier permet de se demander s'il ne s'agit pas d'un dépôt fluvio-marin (Van den Broeck et Rutot, 1888b) se déversant par des ensellements d'un cordon littoral dans une mer encore proche. Toutefois, il n'existe que des traces éparses de cailloutis Onx jusqu'au rivage miocène le plus proche, voire jusqu'à la Gette par laquelle de Heinzelin (1963) prolonge la Meuse de Dinant. La pente longitudinale de cette Meuse de Dinant devait être très faible, car de larges méandres de ce cours d'eau qui se sont recoupés en cours d'incision, devaient exister à ce niveau (Anhée, Annevoie-Rouillon, Profondeville) ; ceux de Chooz et de Revin sont toujours 


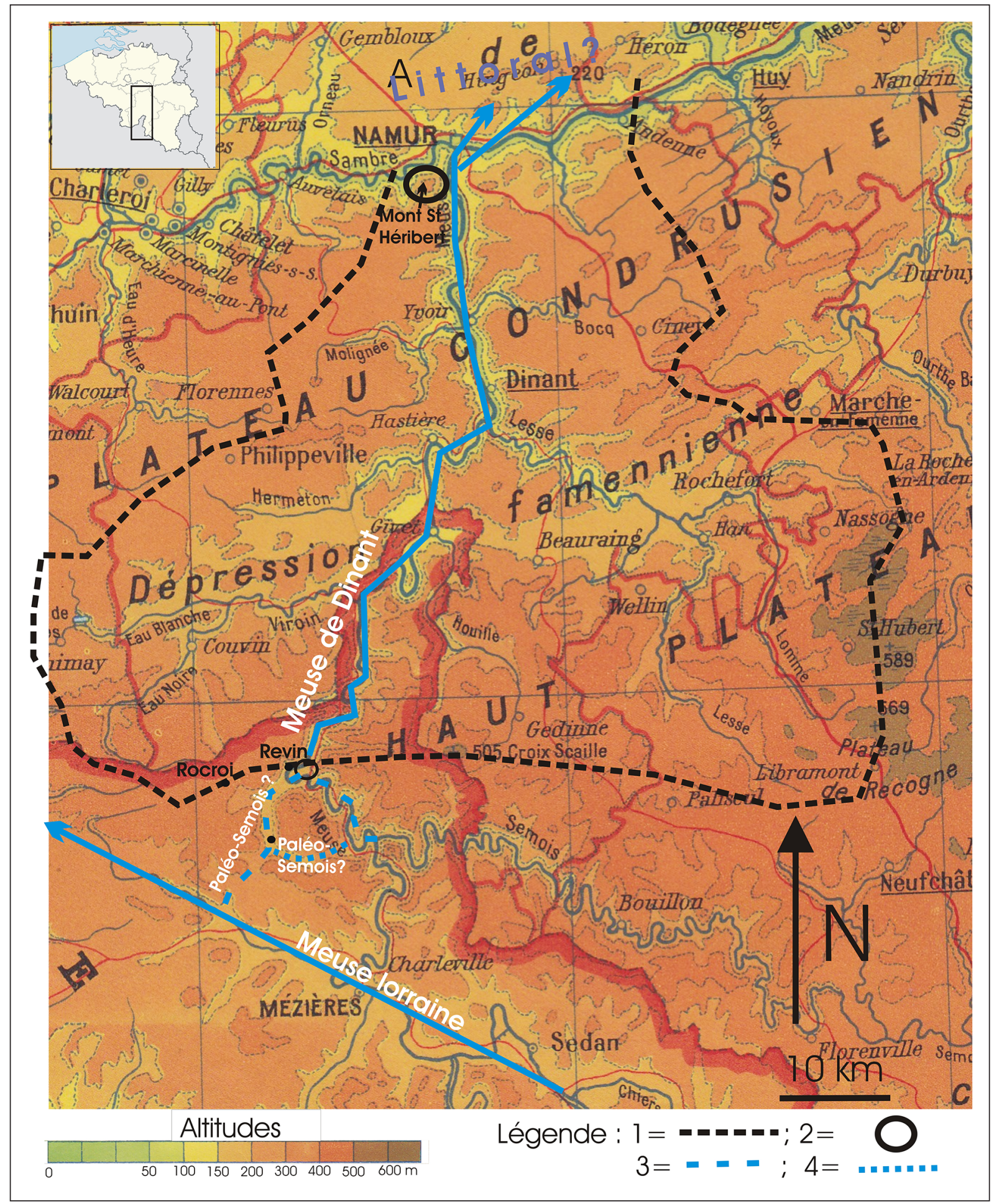

Figure 15. Le bassin de la Meuse de Dinant dans la situation immédiatement antérieure à la capture de la paléo-Semois selon nous (Fond de carte de l'atlas P. Mantnieks, 1955). Le tracé de la Meuse lorraine est ici caricatural, il ne tient compte que du consensus qui existe sur son prolongement par la vallée de la Sormonne. Légende : 1=limite du Bassin de la Meuse de Dinant ; 2= cailloutis Onx du plateau du Mont Saint-Héribert ; 3= paléo-Semois, possible avec capture à Revin selon nous ; 4= paléo-Semois possible avec capture à Deville selon Pissart et al. (1997a). 


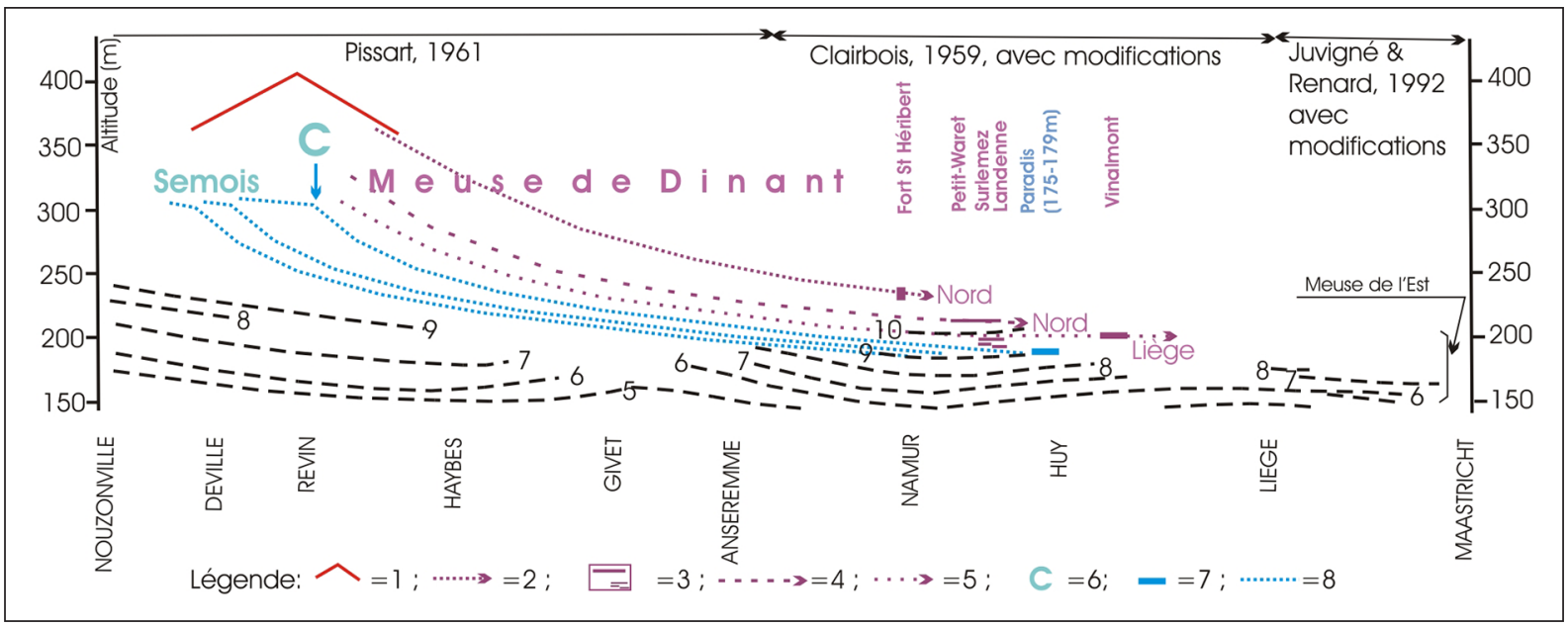

Figure 16. Profils longitudinaux successifs de la Meuse de Dinant et de la terrasse issue de la capture de la Semois. Explications : le fond graphique en noir est un extrait du modèle de Pissart et al. (1997, Fig. 2, p.269) qui sont les auteurs des «modifications ». Légende : 1= position de la crête du Massif de Rocroi ; 2= la Meuse de Dinant primitive passant par le dépôt Onx du Mont Saint-Héribert (Namur ; Van den Broeck et Rutot, 1988b) et poursuivant son cours vers le rivage diestien (de Heinzelin, 1963) ; $3=$ lbxt de cailloutis Onx revisités entre Namur et Andenne (cf. supra) ; 4= la Meuse de Dinant passant par les dépôts Onx de plateau de Petit-Waret et de Surlemez et poursuivant son cours vers le rivage diestien ; $5=$ la Meuse de Dinant au niveau des replats à cailloutis Onx passant le dépôt de Vinalmont et poursuivant son cours vers Liège $; 6=$ position du site de capture de la Semois par la Meuse de Dinant à Revin plutôt qu'à Deville ; $7=1 \mathrm{bt}$ de Paradis/Couthuin avec cailloutis q2m provenant de la Semois ; $8=$ profils longitudinaux successifs de la Meuse et de la Semois inférieure en conséquence de la capture de la Semois.

actifs. Le cailloutis Onx le plus élevé du plateau de Champion est entre 205 et $208 \mathrm{~m}$, soit une dizaine de mètres plus bas que celui de Petit-Waret, mais il repose sur des roches calcaires, si bien que l'on peut attendre qu'il ait été affecté par la karstification (cf. la carrière de Namèche). Dans cette mesure, on ne peut exclure que les trois cailloutis de plateau précités fassent partie d'une même nappe.

\section{Incision de la Meuse de Dinant et ébauche de la vallée Namur-Huy}

Dès le moment où l'incision de la Meuse de Dinant est passée sous l'altitude du toit du cailloutis Onx à Surlemez et à Petit-Waret, 1'évolution de la vallée a eu lieu uniquement sur la partie méridionale du plateau, et le cours d'eau n'a pu se diriger que vers l'Est, au moins depuis Namur jusqu'à Couthuin-Andenne ; on peut voir là l'ébauche du tronçon mosan Namur-Liège. À l'est de la Mehaigne sur le plateau de Vinalmont, le toit du cailloutis $O n x$ le plus élevé se trouve à $203 \mathrm{~m}$, si bien qu'à cette altitude la Meuse de Dinant pouvait à nouveau se déverser dans une mer supposée proche, car l'altitude de la crête de Hesbaye ne dépasse nulle part 203 m entre Vinalmont et Liège. Par ailleurs, en rappelant que la granularité des graviers $O n x$ de replats est un peu plus grossière que celle des graviers de plateau, on peut y voir un léger accroissement de la compétence de la Meuse de Dinant au cours de son incision ; cela pourrait s'expliquer par le déversement de la paléo-Sambre dans la Meuse de Dinant à Namur qui a bien dû avoir lieu, mais il n'existe aucune étude qui fasse état ni des circonstances de cet événement, ni du moment auquel il a eu lieu.

\section{La capture de la Semois enregistrée à Paradis/ Couthuin à 175-179 m}

Après une incision de 33 m (216 m à Petit-Waret ; 183 m à Landenne), le cailloutis de Landenne à $183 \mathrm{~m}$ reste de type $O n x$, et $2 \mathrm{~km}$ plus loin à Paradis/ Couthuin à 175-179 m, le cailloutis est fondamentalement différent : (1) la granularité du gravier est nettement plus grossière ; (2) les quartzites et les grès prennent une part dominante dans le spectre de la nature des cailloux roulés ; (3) les quartz à émoussé sommaire concurrencent les galets de quartz; (4) l'indice d'arrondi de Wadell est nettement plus faible.

Les apports juvéniles dans le cailloutis de Paradis, provenant des roches de haute Ardenne incitent à les attribuer à la capture de la Semois par la Meuse de Dinant. Il n'y a effectivement pas d'autre alternative apparente : (1) si même on ne doit pas perdre de 

entre Namur et Huy (Belgique) à la suite de la capture de la Semois

vue que la Sambre dans sa gestation (de Heinzelin, 1963) a dû rejoindre la Meuse à un niveau encore indéterminé, ses affluents ne drainaient pas davantage que maintenant les roches pré-ordovicennes de l'actuelle Entre-Sambre-et-Meuse ; (2) dans la mesure où la capture de la Meuse lorraine fut nécessairement postérieure à celle de la Semois (Pissart, 1961 et suivants), il est difficile d'attribuer, cet apport soudain à l'un ou l'autre affluent qui draine le flanc méridional du Massif pré-calédonien et qui impliquerait dans l'ordre chronologique les captures successives de la Lesse (Massif de Recogne), de la Houille (Croix Scaille) puis du Viroin (Massif de Rocroi) ; (3) enfin l'entrée en scène dans les cailloutis mosans entre 110 et $120 \mathrm{~m}$ (voir plus haut) indique que l'on peut envisager que la capture de la Meuse lorraine soit postérieure à la T. de Paradis/ Couthuin, ce qui est discuté par Juvigné et Houbrechts (ce fascicule).

\section{DISCUSSION}

\section{A. À propos des niveaux auxquels les captures de la Semois et de la Meuse lorraine ont eu lieu}

Parmi les auteurs qui se sont interrogés sur la capture de la Meuse lorraine par la Meuse de Dinant, Rutot (1897), Dollfus (1900) et Cornet (1903) l'ont placée dans les altérites du socle paléozoïque du Massif de Rocroi, c'est-à-dire de façon implicite à l'altitude actuelle du plateau (400 m). Rutot y voit une relation avec l'affaissement de l'Ardenne lié à la transgression miocène. Même si ces auteurs ne citent pas la capture de la Semois, il est implicite que celle-ci doit avoir eu lieu pratiquement au même niveau que celle de la Meuse lorraine. Le cours d'eau capturant ne peut être que la Meuse de Dinant primitive dont les cailloutis Onx les plus élevés (au Fort Saint-Héribert/Namur-S à $235 \mathrm{~m}$ ) seraient un témoin (Figure 16).

Pissart (1961, 1975), puis Pissart et al. (1997) ont situé les deux captures précitées à $300 \mathrm{~m}$ d'altitude et ont placé celle de la Meuse lorraine dans la transition mio-pliocène. Sur le modèle de Pissart et al. (1997), on lit que le lbt de Paradis/Couthuin qui a reçu les premières alluvions de la Semois fait partie de la terrasse T9 (Figure 16). À l'amont, le tronçon de cette même terrasse T9 entre Charleville et Haybes pourrait être l'aboutissement de la vague d'érosion régressive qui s'est propagée dans la vallée de la Semois et qui allait amputer le bassin supérieur de la Chiers (Pissart, 1961). À l'aval de Huy, la terrasse T9 n'a pas de lbxt connu avant le Limbourg néerlandais méridional où elle doit nécessairement se trouver au-dessus de T8, et de ce fait dans la vallée abandonnée nommée Ostmaas (Hol, 1949).

\section{B. À propos des modèles de terrasses mosanes impliqués ici}

Le commentaire qui suit est évidemment limité aux terrasses depuis l'Onx de plateau jusqu'au lbt de Paradis/Couthuin sur le seul tronçon revisité. Parmi tous les modèles publiés (voir Juvigné et al., 2020a), nous n'en retenons ici que deux, celui produit par Clairbois $(1957,1959)$ pour le tronçon d'Anseremme jusqu'à Liège et le dernier modèle de synthèse en date (Pissart et al., 1997) parce qu'il a intégré celui précité de Clairbois.

Le modèle de Clairbois $(1957,1959)$ est construit par la méthode du raccord de proche en proche d'environ un millier de replats (avec cailloutis, dénudés, ou lambeaux d'aplanissement partiel). Dans le tronçon et la tranche de dénivelée qui nous occupent, trois terrasses ont été reconstituées. À partir de la plus haute, elles portent les labels T9, T9' et T8, et elles sont présentées en contre-pente à l'aval de Champion (Figure 17). Ce travail détaillé a été suivi d'une publication formelle (Clairbois, 1959), mais c'est évidemment dans le premier que l'on trouve les observations de terrain qui permettent 1'analyse critique résumée ci-dessous, mais discutée en détails dans Juvigné et al. (2020a).

D'une analyse critique détaillée lbt par lbt, du modèle de Clairbois (1957), il ressort que les raccords proposés par l'auteur ne sont absolument pas soutenus par les observations de terrain et que la méthode du raccord de proche en proche qui y est appliquée part du principe que le basculement tectonique proposé par Fourmarier (1924) et confirmé par Mouchamps $(1927,1933)$ était une réalité acquise. Dans le même esprit, Pissart (1975) travaillant sur les données originales de Clairbois (1957) a amplifié le basculement précité, pour en faire le flanc occidental d'un anticlinal dont la retombée orientale est située dans la région liégeoise. Les détails de cette analyse critique sont accessibles dans le rapport de travail de Juvigné et al. (2020a) ; il y est démontré que le tracé des hautes terrasses repose sur des observations et une méthodologie 


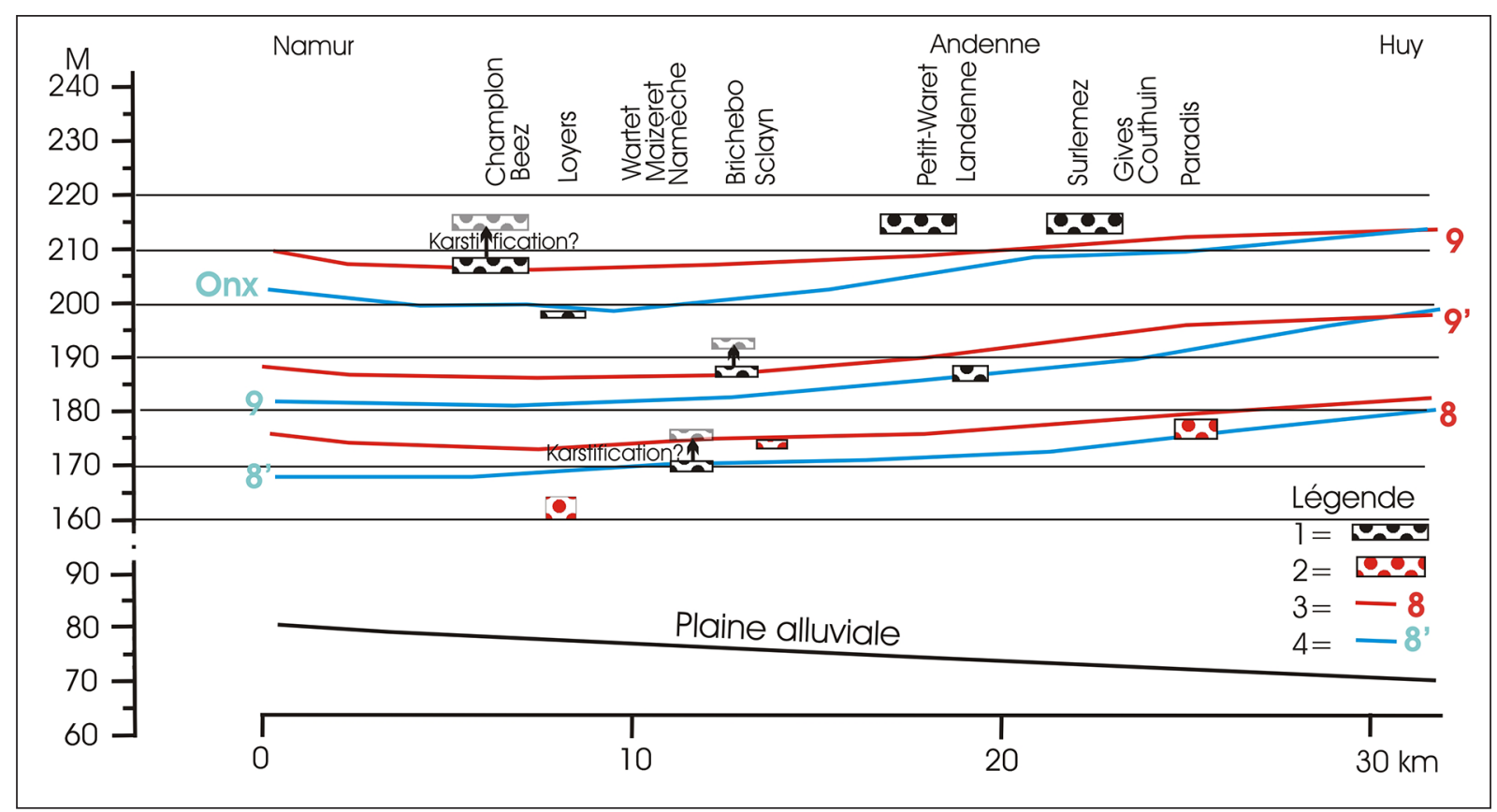

Figure 17. Deux modèles de profils longitudinaux de très hautes terrasses mosanes sur la base des seules données de Clairbois (1957). Légende : 1= position de cailloutis Onx d'après nos travaux ; $2=$ position de cailloutis q2m d'après nos travaux ; $3=$ profils et labels de terrasses selon Clairbois (1957) ; 4= profils et labels de terrasses selon Pissart et al. (1997). Explications : les profils longitudinaux sont des extraits des modèles originaux de terrasses qui ont été scannés et adaptés à une échelle x-y commune.

qui ne justifient en rien de leur avoir attribué une contre-pente. Cette contre-pente a déjà été formellement infirmée entre Seilles et Huy (Juvigné et al., 2013). Par ailleurs, la Figure 17 montre que les profils respectifs ne passe pas systématiquement par les lbxt dont nous avons déterminé la position avec des outils autres que la lecture sur des cartes topographiques. Les modèles de hautes terrasses mosanes sont donc à revoir dans l'ensemble du modèle de référence de Pissart et al. (1997).

\section{C. À propos de l'âge des très hautes terrasses mosanes}

D'une part, en matière de chronologie des terrasses, les sites de référence se trouvent essentiellement dans le Limbourg néerlandais (Felder et Bosch, 1989 ; Van den Berg, 1996). D'autre part, entre Charleville et le Limbourg, on se heurte de toute évidence à la fiabilité du modèle de Pissart et al. (1997a), car : (1) Juvigné et al. (2013) ont montré que la terrasse dite principale de la Meuse (T4) qui est rapportée en contre-pente dans la littérature (Clairbois, 1957 ; 1959 ; Pissart, 1975, Pissart et al., 1997a) présente en réalité une pente identique à celle de la plaine d'inondations, au moins entre Seilles et Huy; (2) Juvigné et al. (2020a) montrent plus haut que les déformations tectoniques attri- buées aux hautes terrasses entre Namur et Huy sont en contradiction avec les données pétrographiques propres aux cailloutis des terrasses ; (3) l'étude récente de très hautes terrasses à Bois de Breux (Liège) soulève l'hypothèse d'un basculement transversal de la vallée de la Meuse liégeoise (Juvigné et Van Campenhout, 2020). Indépendamment des réserves précitées, si l'on suit le modèle de Pissart et al. (1997) tel qu'il se présente, la terrasse de Paradis/Couthuin (T9) débouche dans l'Ostmaas au niveau de la T. de Kosberg, mais celle-ci fait partie de la série des terrasses à petits galets de quartz. Dans cette même vallée abandonnée, la première terrasse à cailloux ardennais est la T. de Noorbeek dont l'âge est estimé entre 1,9 et 1,95 Ma par Felder et Bosch (1989) et à 2,44 Ma par Van den Berg (1996). Indépendamment de toutes les incertitudes évoquées ci-dessus, il semble que l'on puisse retenir que la T. de Paradis/Couthuin remonte à la première moitié du Pléistocène inférieur dont les limites formelles sont actuellement 2,58 Ma et 0,781 Ma.

\section{CONCLUSIONS}

Dans la région de Namur, les cailloux Onx présentent un émoussé qui atteste leur façonnement sur des plages marines probablement tongriennes (Oligocène inférieur). Ils ont été repris en charge par 

entre Namur et Huy (Belgique) à la suite de la capture de la Semois

la Meuse de Dinant, et ont été accumulés en milieu sub-littoral dans une tranche de dénivelée comprise entre $235 \mathrm{~m}$ (Mont Saint-Héribert) et le plateau de Hesbaye namuroise (Petit-Waret et Surlemez : 213$217 \mathrm{~m}$ ) pendant une période indéterminée entre la régression oligocène et la transgression miocène.

L'incision de la Meuse de Dinant a eu lieu uniquement sur la retombée méridionale du plateau où une série de replats en gradins ont été façonnés, chacun d'entre eux est tapissé de cailloutis Onx en épaisseur pluri-décimétrique. Au-dessus de 203 m, l'écoulement s'est fait nécessairement au moins jusqu'à Vinalmont (lez Huy), et entre 203 et 200 m, il était nécessairement contraint d'atteindre Liège (nappe de Rond Péry-Fontaine en bout de piste de Bierset airport). Ce n'est qu'à partir de cette dernière situation que l'existence de la Traînée mosane existe.

C'est à l'altitude de 180 m à Couthuin (lez Huy) sur socle schisto-gréseux (Houiller) que le lit de la Traînée mosane a été subitement envahi par un cailloutis $q 2 m$ attribué à la capture de la Semois survenue à Revin (France). Le même changement pétrographique est enregistré à Namur entre 165 et $168 \mathrm{~m}$ sur socle de roches solubles (calcaire et dolomie), si bien que dans cette dernière région, on peut hésiter entre des effondrements karstiques, ou un basculement tectonique du tronçon impliqué.

\section{REMERCIEMENTS}

Nous adressons nos chaleureux remerciements aux entreprises Sagrex à Beez et Lhoist à Namèche qui nous ont assistés dans le prélèvement de cailloutis au niveau des découvertures de leur carrière, et à Monsieur Jacky Wéra, agriculteur à Paradis/Couthuin qui nous a offert toutes les facilités pour nos diverses tâches destinées à faire du lbt de la terrasse qu'il cultive, un site géomorphologique de référence.

\section{BIBLIOGRAPHIE}

Bellière, M. (1924-1925). Un nouveau gisement d'Onx de la planchette de Malonne. Annales de la Société géologique de Belgique, XLVIII, 94-97.

Bestel, A. (1949). Les terrasses de la Meuse et de la Semois. La cuvette d'Haulme et la plaine de Lumes. Bulletin de la Société d'Histoire naturelle des Ardennes, 36-42.

Bourguignon, P. (1954). Les sables des Hautes Fagnes. Annales de la Société géologique de Belgique, 77, B201-241.
Briquet, A. (1907). La vallée de la Meuse en aval de Liège. Bulletin de la Société belge de Géologie, de Paléontologie et d'Hydrographie, 11, 347-364.

Briquet, A. (1922). Le Néogène du nord de la Belgique et des Pays-Bas et ses relations stratigraphiques. Bulletin de la Société belge de Géologie, de Paléontologie et d'Hydrologie, XXXII, G9-91.

Bustamante-Santa Cruz, L. (1973). Les minéraux lourds des alluvions sableuses du bassin de la Meuse. Thèse de doctorat, Katholieke Universiteit te Leuven, Faculteit der Wetenschappen, 355 p., Leuven.

Clairbois, A.-M. (1957). L'évolution du cours de la Meuse entre Liège et Anseremme au cours du Quaternaire. Université de Liège, Laboratoire de Géographie physique. Mémoire de licence, $175 \mathrm{p}$.

Clairbois, A.-M. (1959). L'évolution du cours de la Meuse entre Liège et Anseremme au cours du Quaternaire. Annales de la Société géologique de Belgique, 82, B213-233.

Cornet, J. (1903). Etudes sur l'évolution des rivières belges. Annales de la Société géologique de Belgique, XXXI, M 261-500.

De Heinzelin, J. (1963). Le réseau hydrographique de la région gallo-belge au Néogène. Essais de reconstitution. Bulletin de la Société belge de Géologie, de Paléontologie et d'Hydrologie, 72, 137-148.

Demoulin, A. (1987). Les sables oligocènes du Plateau des Hautes Fagnes : une synthèse. Bulletin de la Société belge de Géologie, de Paléontologie et d'Hydrographie, 96, 81-90.

Demoulin, A. \& Hallot, E. (2009). Shape and amount of the Quaternary uplift of the western Rhenish shield and the Ardennes (western Europe). Tectonophysics, 474, 696-708.

Dewez, T. (1998). Influences néotectoniques sur la géomorphologie de la région de Huy : le méandre de Leumont et la butte d'Ombret. Bulletin de la Société géographique de Liège, 35, 19-27.

Dollfus, G. (1900). Relation entre la structure géologique du Bassin de Paris et son hydrographie. Annales de Géographie, IX, 313-339.

Felder, W.M. \& Bosch, P.W. (1989). Geologische kaart van Zuid-Limburg en omgeving. Afzettingen van de Maas. Rijks Geologische Dienst, Haarlem, NL.

Forir, H. (1897-1898). Quelques mots sur les dépôts tertiaires de l'Entre Sambre et Meuse. Annales de la Société géologique de Belgique, XXV, M 33-39.

Fourmarier, P. (1905). Le cours de la Meuse aux environs de Huy. Annales de la Société géologique de Belgique, 34, M 219-236.

Fourmarier, P. (1924). Les dernières ondulations du sol et les terrasses de la Meuse. Annales de la Société géologique de Belgique, livre jubilaire, pp. 110-113.

Hacken, M. (1948). De Maasvallei Tussen Agimont en Namen. Morphologische Studie. Thèse inédite, Université Catholique de Louvain.

Hol, J., 1949. Geomorfologie. Handboek der Geographie van Nederland, I, 240-319. 
Juvigné, E., Cordy, J.-M., Demoulin, A., Geeraerts, R., Hus, J. \& Renson, V. (2005). Le site archéo-paléontologique de La Belle-Roche (Belgique) dans le cadre de l'évolution géomorphologique de la vallée de l'Amblève inférieure. Geologica Belgica, 8/1-2, 121-133.

Juvigné, E., Dimodica, K., Houbrechts, G. \& Pirson, S. (2013). La pente longitudinale de la terrasse principale de la Meuse revisitée de Seilles à Huy (Belgique). Bulletin de la Société géographique de Liège, 61, 69-80.

Juvigné, E., Demoulin, A., Houbrechts, G. \& Paulissen, E. (2015). Des terrasses de fond de vallée de la Meuse dans la région de Huy (Belgique). Bulletin de la Société géographique de Liège, 65, 27-50.

Juvigné, E. \& Houbrechts, G. (2020). Remise en question de l'âge de la capture de la Meuse lorraine à la lumière de la répartition des amphiboles des Vosges dans les terrasses mosanes. Bulletin de la Société géographique de Liège, ce fascicule.

Juvigné, E., Houbrechts, G. \& Van Campenhout, J. (2020a). À propos d'un changement pétrographique majeur dans les terrasses de la Meuse entre Namur et Huy (Belgique) : de la Meuse de Dinant à la capture de la Semois. Rapport d'activité sur le site institutionnel de l'ULiège : http://hdl.handle.net/2268/244521

Juvigné, E., Hatert, F. \& Bruni, Y. (2020b). À propos de la détermination des chloritoïdes en frottis : le cas des alluvions de la Meuse et d'affluents ardennais. Rapport d'activité sur le site institutionnel de l'ULiège : http://hdl.handle.net/2268/245521

Lohest, M. (1896). Les dépôts tertiaires de l'Ardenne et du Condroz. Annales de la Société géologique de Belgique. XVII, 37-59.

Lorié, J. (1919). Le Diluvium ancien de la Belgique et du Nord de la France. Annales de la Société géologique de Belgique, 62, M 221-409.

Macar, P. (1945). La valeur, comme moyen de corrélation, des cailloux d'oolithe silicifiée et l'origine des graviers dits «Onx » des Hautes-Fagnes. Bulletin de la Société belge de géologie, de Paléontologie et d'Hydrologie, LIV, 214-253.

Macar, P. \& Meunier, J. (1955). La composition lithologique de la « Traînée mosane » et ses variations. Annales de la Société géologique de Belgique, 78, B 63-87.

Meunier, J. (1953). La "Traînée mosane »-Quelques résultats de recherches. Étude de géographie physique. Université de Liège. Travail de fin d'études, inédit, $55 \mathrm{p}$.

Mouchamps, L. (1927). Les terrasses de la Meuse et de la Sambre. Université de Liège, Laboratoire de Géographie, Thèse de Doctorat, inédite, 133 pages.

Mouchamps, L. (1933). Les terrasses de la Meuse et de la Sambre. Annales de la Société géologique de Belgique, 82, B 213-233.

Pissart, A. (1961). Les terrasses de la Meuse et de la Semois. La capture de la Meuse de la Meuse lor- raine par la Meuse de Dinant. Annales de la Société géologique de Belgique, 84, 1-108.

Pissart, A. (1975). La Meuse en France et en Belgique. Formation du bassin hydrographique. Les terrasses et leurs enseignements. In, L'évolution quaternaire des bassins fluviaux de la Mer du Nord méridionale, Centenaire de la Société géologique de Belgique, 1974, 105-131.

Pissart, A., Harmand, D. \& Leendert, K. (1997). L'évolution du cours de la Meuse de Toul à Maastricht depuis le Miocène: corrélations chronologiques et traces des captures de la Meuse lorraine d'après les minéraux denses. Géographie physique et Quaternaire, 51, 267-284.

Rigo, M. (1935). Etudes des terrasses fluviales sur le versant sud de l'Ardenne. Annales de la Société géologique de Belgique, 59, 1-30.

Rixhon, G. \& Demoulin, A., (2018). The Picturesque Ardennian Valleys: Plio-Quaternary Incision of the Drainage System in the Uplifting Ardenne. In Demoulin A. (ed), Landscapes and Landforms of Belgium and Luxembourg, Springer, 159-175.

Roussillon, T., Piégay, H., Sivignon, I., Tougne, L. \& Lavigne, F. (2009). Automatic computation of pebble roundness using digital imagery and discrete geometry. Computers \& Geosciences, 35, 1992-2000.

Rutot, A. (1897). Les origines du Quaternaire de la Belgique. Bulletin de la Société belge de Géologie, de Paléontologie et d'Hydrologie, XI, 1-140.

Rutot, A. (1900). Carte géologique de la Belgique, $\mathrm{n}^{\circ} 145$, Andenne, planchette 1-2, Archives du Service géologique de Belgique, note SvG-270.

Rutot, A. (1908). Sur l'âge des dépôts connus sous les noms de sable de Mol, d'argile de Campine, de cailloux de quartz blanc, d'argile d'Andenne et de sable à faciès marin noté $\mathrm{Om}$ dans la légende de la carte géologique de la Belgique au 40.000e. Académie royale de Belgique, Classe des Sciences, Mémoire, 2e série, t. II.

Stainier, X. (1894). Le cours de la Meuse depuis l'ère tertiaire. Bulletin de la Société belge de Géologie, de Paléontologie et d'Hydrologie, VIII, M 83-101.

Stainier, X. (1901a). Carte géologique de la Belgique, $\mathrm{n}^{\circ} 145$, Andenne, planchette 1-2.

Stainier, X. (1901b). Carte géologique de la Belgique, $\mathrm{n}^{\circ} 144$, Namur, planchette 3-4.

Stainier, X. (1926). Histoire de la Meuse quaternaire dans les environs de Huy. Annales de la Société scientifique de Bruxelles, 46, 272-285.

Stevens, Ch. (1922). Sur d'anciens tracés de rivières aux environs de Mézières. Bulletin de la Société belge de Géologie, de Paléontologie et d'Hydrologie, 32, 28-32.

Van den Berg, M.W. (1996). Fluvial sequences of the Maas: a 10 Ma record of neotectonics and climate change at various time-scales. Ph.D. Thesis, University of Wageningen, The Netherlands. 
Van Straaten, L.M.J.U. (1946). Grindonderzoek in Zuid-Limburg. Medel. Géol. Stichting, ser. C, VI, $\mathrm{n}^{\circ} 2,146 \mathrm{p}$.

Van Straaten, L.M.J.U. (1947). Étude quantitative des graviers de la Meuse. La Géologie des terrains récents dans l'ouest de l'Europe, Bruxelles, pp. 142-151.

Van den Broeck, E. (1889). Les cailloux oolithiques des graviers tertiaires des hauts-plateaux de la Meuse. Bulletin de la Société belge de Géologie, de Paléontologie et d'Hydrologie, III, 404-411.

Van den Broeck, E. \& Rutot, A. (1888a). De l'extension des sédiments tongriens sur les plateaux du Condroz, et de l'Ardenne et du rôle géologique des vallées d'effondrement dans les régions à zones calcaires de la Haute-Belgique. Bulletin de la Société belge de Géologie, de Paléontologie et d'Hydrologie, II, 9-25.

Van den Broeck, E. \& Rutot, A., (1888b). Deuxième note sur la reconnaissance géologique et hydrologique des emplacements des forts de la Meuse. Bulletin de la Société belge de Géologie, de Paléontologie et d'Hydrologie, 1888, 81-90.

Vandenberghe, N. (2017). Tectonic and climatic signals in the Oligocene sediments of the Southern NorthSea Basin. Geologica Belgica, 20/3-4, 105-123.

Wadell, H. (1932). Shape and roundess of rock particles. Journal of Geology, 40, 443-451.
Wadell, H. (1933). Sphericity and roundess of rock particles. Journal of Geology, 41, 325-331.

Carte topographique de la Belgique, 1904, Institut géographique national, Bruxelles.

Atlas P. Mantnieks, Institut cartographique P. Mantnieks, Bruxelles, 1955.

Coordonnées des auteurs :

Étienne JUVIGNÉ

ULiège Département de Géographie Unité de Géographie physique ejuvigne@skynet.be

Geoffrey HOUBRECHTS

ULiège Département de Géographie Unité de Géographie physique - UR Sphères G.Houbrechts@uliege.be Jean VAN CAMPENHOUT ULiège Département de Géographie Unité de Géographie physique - UR Sphères jean.vancampenhout@uliege.be 
